\title{
TITLE:
}

\section{Parametric instability of classical Yang-Mills fields in an expanding geometry}

\section{$\operatorname{AUTHOR}(\mathrm{S})$ :}

Tsutsui, Shoichiro; Kunihiro, Teiji; Ohnishi, Akira

\section{CITATION:}

Tsutsui, Shoichiro ...[et al]. Parametric instability of classical Yang-Mills fields in an expanding geometry. Physical Review D 2016, 94(1): 016001.

ISSUE DATE:

2016-07-01

URL:

http://hdl.handle.net/2433/233193

RIGHT:

(C) 2016 American Physical Society. 
PHYSICAL REVIEW D 94, 016001 (2016)

\title{
Parametric instability of classical Yang-Mills fields in an expanding geometry
}

\author{
Shoichiro Tsutsui, ${ }^{1, *}$ Teiji Kunihiro, ${ }^{1}$ and Akira Ohnishi ${ }^{2}$ \\ ${ }^{1}$ Department of Physics, Faculty of Science, Kyoto University, Kyoto 606-8502, Japan \\ ${ }^{2}$ Yukawa Institute for Theoretical Physics, Kyoto University, Kyoto 606-8502, Japan
}

(Received 21 January 2016; published 18 July 2016)

\begin{abstract}
We investigate the instability of a classical Yang-Mills field in an expanding geometry under a color magnetic background field within the linear regime. We consider homogeneous, boost-invariant, and timedependent color magnetic fields simulating the glasma configuration. We introduce the conformal coordinates which enable us to map an expanding problem approximately into a nonexpanding problem. We find that the fluctuations with finite longitudinal momenta can grow exponentially due to parametric instability. Fluctuations with finite transverse momenta can also show parametric instability, but their momenta are restricted to be small. The most unstable modes start to grow exponentially in the early stage of the dynamics, and they may affect the thermalization in heavy-ion collisions.
\end{abstract}

DOI: 10.1103/PhysRevD.94.016001

\section{INTRODUCTION}

Recent developments in physics of high-energy heavyion collisions have unveiled the property of the quark-gluon plasma (QGP) and have raised a puzzle in the preequilibrium dynamics before the QGP formation. High-energy heavy-ion collision experiments have been performed extensively at the Relativistic Heavy-Ion Collider (RHIC) at Brookhaven National Laboratory and LHC at CERN. Theoretical analyses of data based on hydrodynamical approaches are found to be successful in explaining various observables such as hadron momentum spectra and collective flows in the nucleus-nucleus collisions [1-3]. The hydrodynamical analyses suggest that QGP formed at the RHIC and LHC would be a strongly interacting fluid rather than a weakly interacting gas. However, it should be noted that the hydrodynamical models still pose a puzzle. The early thermalization required by the hydrodynamical analyses appears inconsistent with perturbative QCD results, such as those given by the bottom-up scenario [4,5]. To resolve the puzzle, we need deeper understanding of the dynamical nature of the initial stage of the created matter, say, in view of far-from-equilibrium dynamics of nonAbelian gauge theory.

The initial stage of relativistic heavy-ion collisions may be well described by the effective theory based on the notion of the color glass condensate [6,7]. It is shown that the longitudinal color-flux tubes which consist of both color electric and magnetic fields should be formed right after the collision of two nuclei. This state with the longitudinal color-flux tube is called glasma [8]. The time evolution of the low-energy sector is well described by classical Yang-Mills (CYM) theory as the first approximation although quantum fluctuations can significantly affect

tsutsui@ruby.scphys.kyoto-u.ac.jp the dynamics. For instance, quantum fluctuations would trigger plasma instabilities, which in turn might cause the emergence of chaoticity, turbulent spectra, rapid particle production, and thermalization [9-12].

Glasma instabilities in heavy-ion collisions have been extensively discussed. Among them, longitudinal fluctuations are found to cause an instability in glasma [13-18]. One possible underlying mechanism of the instabilities is the non-Abelian analog of the Weibel instability [19-22], which leads to an exponential amplification of the color magnetic field and current in anisotropic systems. The longitudinal color magnetic field may also induce the Nielsen-Olesen instability [23-28], which is the exponential growth of gauge fluctuations caused by the anomalous Zeeman effect in spin-1 systems.

Recently, it has been suggested that the CYM field under a time-dependent homogeneous color magnetic background field shows instability in a nonexpanding geometry [29]. It has been clarified that the origin of the instability is parametric instability and the CYM field in a homogeneous background has multiple instability bands extending to the transverse as well as the longitudinal momentum directions [30]. The parametric instability is ubiquitous in physics from classical mechanical problems on a pendulum to quantum field theories [31], as well as in the cosmic inflation [32]. These results suggest that the parametric instability may also play an essential role in the thermalization process in heavy-ion collisions.

From a phenomenological point of view, it is natural to ask ourselves whether and how the parametric instability found in a nonexpanding geometry persists in an expanding geometry. In general, background fields decrease in time due to the expansion, so one may expect that the instability would disappear or at least become less significant than in the nonexpanding geometry. However, the detailed studies of a scalar field theory suggest that the parametric 
instability can keep significance even in an expanding geometry [33,34].

In this article, we investigate the instability of the CYM field in an expanding geometry in the linear regime. Specifically, we focus on the system with the longitudinal color magnetic field which is homogeneous, boost invariant, and time dependent. We also assume the one-dimensional Bjorken expansion. The background field considered here damps due to the expansion and at the same time oscillates in time. We introduce a natural chronological variable called conformal time which enables us to map an expanding problem into a nonexpanding problem. Then, the relevant equation is found to have a form of a temporally periodicdriven equation like $\ddot{f}+p^{2} f+\lambda \mathrm{cn}^{2}(t ; k) f=0$. This equation involves the Jacobi elliptic function $\mathrm{cn}(t ; k)$ and is called Lamé's equation for a constant momentum $p$ and a constant coefficient $\lambda$. Lamé's equation shows an exponential instability at $\lambda=-1$ and 2 for a small momentum $p$. In the expanding case, the equation of motion is found to contain an effective momentum, which is a function of the initial momentum and the conformal time and has decreasing longitudinal and increasing transverse components in time. As a result, fluctuations with finite longitudinal momenta tend to be unstable due to the parametric instability, while those with a finite transverse momentum can also show instability but their momenta are restricted to small values.

This article is organized as follows. In Sec. II, we introduce the conformal time and derive equations of motion of a CYM field in an expanding geometry. We also discuss properties of a homogeneous, boost-invariant, and time-dependent background field. In Sec. III, we give a brief outline of the Floquet theory and the instability band structure of Lamé's equation as a useful tool to understand the expanding problem. In Sec. IV, we show the results of the linear stability analysis. Finally, we summarize our results and give concluding remarks in Sec. V. In Appendix A, we show the explicit form of the linearized equation of motion of fluctuations. In Appendix B, we comment on the origin of a linearly divergent solution. In Appendix $\mathrm{C}$, we give a quantitative discussion on the growth rate.

\section{CLASSICAL YANG-MILLS FIELD IN AN EXPANDING GEOMETRY}

In this section, we derive the equation of motion (EOM) of the CYM field in an expanding geometry under a color magnetic background field, which is homogeneous, boost invariant, and time dependent. In Sec. II A, we introduce the conformal time for the sake of mapping the expanding problem into a nonexpanding problem. In Sec. II B, we discuss properties of the background field. In Sec. II C, we show an explicit form of the linearized EOM of fluctuations.

\section{A. Expanding geometry and conformal time}

We introduce the proper time $\tau$ and the space-time rapidity $\eta$ to describe boost-invariant longitudinal expansion. They are defined by

$$
\begin{gathered}
\tau=\sqrt{t^{2}-z^{2}}, \\
\eta=\frac{1}{2} \log \frac{t+z}{t-z} .
\end{gathered}
$$

The action of the CYM field in the $\tau-\eta$ coordinate is given by

$$
S=\int d \tau d^{2} x_{\perp} d \eta \sqrt{-g}\left(-\frac{1}{4} g^{\mu \nu} g^{\lambda \sigma} \mathcal{F}_{\mu \lambda}^{a} \mathcal{F}_{\nu \sigma}^{a}\right) .
$$

Here, $\quad \mathcal{F}_{\mu \nu}^{a}=\partial_{\mu} \mathcal{A}_{\nu}^{a}+\partial_{\nu} \mathcal{A}_{\mu}^{a}+f^{a b c} \mathcal{A}_{\mu}^{b} \mathcal{A}_{\nu}^{c}$ is the field strength tensor, and $f^{a b c}$ is the structure constant. The coupling constant is included in the definition of the gauge field $\mathcal{A}_{\mu}^{a}$. The explicit form of the metric $g^{\mu \nu}$ is given by

$$
\begin{gathered}
g^{\mu \nu}=\operatorname{diag}\left(1,-1,-1,-1 / \tau^{2}\right), \\
g_{\mu \nu}=\operatorname{diag}\left(1,-1,-1,-\tau^{2}\right), \\
\operatorname{det} g_{\mu \nu} \equiv g=-\tau^{2} .
\end{gathered}
$$

Gauss's law in the $\tau-\eta$ coordinate is expressed as

$$
\mathcal{D}_{i} \mathcal{E}_{i}^{a}+\mathcal{D}_{\eta} \mathcal{E}_{\eta}^{a}=0
$$

where the color electric fields are defined as $\mathcal{E}_{i}=\tau \partial_{\tau} A_{i}$ $(i=x, y)$ and $\mathcal{E}_{\eta}=\frac{1}{\tau} \partial_{\tau} A_{\eta}$. The covariant derivative is given by $\mathcal{D}_{\mu}=\partial_{\mu}-i \mathcal{A}_{\mu}$.

It is useful to see how the gauge field decreases by the expansion of the system. The Bjorken solution of the longitudinally expanding hydrodynamics tells us that the energy density decreases as $\epsilon \propto \tau^{-4 / 3}$ [35]. Provided that this behavior also applies to the gauge field, we expect that the amplitude of the gauge field decreases as $\mathcal{A} \propto \tau^{-1 / 3}$, since the energy density of CYM fields contains terms proportional to $\mathcal{A}^{4}$. Motivated by this observation, let us consider the following time-dependent scale transformation [33],

$$
\begin{aligned}
& \partial_{\tau}=\tau^{-1 / 3} \partial_{\theta}, \\
& \mathcal{A}_{i}^{a}=\tau^{-1 / 3} A_{i}^{a}, \\
& \mathcal{A}_{\eta}^{a}=\tau^{-1 / 3} A_{\eta}^{a},
\end{aligned}
$$

where $\mathcal{A}$ and $A$ denote the gauge fields in the $\tau$ - $\eta$ coordinate and in the scaled coordinate, respectively. We introduce the new chronological variable called conformal time $\theta$. The 
PARAMETRIC INSTABILITY OF CLASSICAL YANG- ...

relation between the conformal time and the proper time is explicitly given by

$$
\tau(\theta)=\left(\frac{2}{3} \theta\right)^{3 / 2} \equiv \tilde{\theta}^{3 / 2}
$$

Taking the Schwinger gauge $\mathcal{A}_{\tau}^{a}=0$, the action in the new coordinate reads

$$
\begin{aligned}
S= & \int d \theta d^{2} x_{\perp} d \eta\left[\frac{1}{2}\left(\partial_{\theta} A_{i}^{a}\right)^{2}-\frac{1}{2} \frac{1}{9 \tilde{\theta}^{2}} A_{i}^{a} A_{i}^{a}-\frac{1}{4} \tilde{F}_{i j}^{a} \tilde{F}_{i j}^{a}\right. \\
& \left.+\frac{1}{\tilde{\theta}^{3}}\left(\frac{1}{2}\left(\partial_{\theta} A_{\eta}^{a}\right)^{2}-\frac{1}{2} \frac{7}{9 \tilde{\theta}^{2}} A_{\eta}^{a} A_{\eta}^{a}-\frac{1}{2} \tilde{F}_{\eta i}^{a} \tilde{F}_{\eta i}^{a}\right)\right] .
\end{aligned}
$$

From now on, the capital letters denote all space components as $I, J, \ldots=x, y, \eta$, and the lower letters only transverse components as $i, j, \ldots=x, y$. The field strength tensor and covariant derivative in the new coordinate are defined by

$$
\begin{gathered}
\tilde{F}_{\mu \nu}^{a}=\tilde{\partial}_{\mu} A_{\nu}^{a}+\tilde{\partial}_{\nu} A_{\mu}^{a}+f^{a b c} A_{\mu}^{b} A_{\nu}^{c}, \\
\tilde{D}_{I}^{a b}=\tilde{\partial}_{I} \delta^{a b}+f^{a c b} A_{I}^{c}, \\
\tilde{\partial}_{I}=\tau^{1 / 3} \partial_{I}=\tilde{\theta}^{1 / 2} \partial_{I} .
\end{gathered}
$$

In the action Eq. (12), the overall time dependence coming from the metric is absorbed into the coefficient of the quadratic terms and the spatial derivatives, so that the action has a similar form to that in a nonexpanding geometry. The Euler-Lagrange equation is given by

$$
\begin{aligned}
& \partial_{\theta}^{2} A_{i}^{a}+\frac{1}{9 \tilde{\theta}^{2}} A_{i}^{a}-\frac{1}{\tilde{\theta}^{3}} \tilde{D}_{\eta} \tilde{F}_{\eta i}^{a}-\tilde{D}_{j} \tilde{F}_{j i}^{a}=0, \\
& \partial_{\theta}\left(\frac{1}{\tilde{\theta}^{3}} \partial_{\theta} A_{\eta}^{a}\right)+\frac{7}{9 \tilde{\theta}^{5}} A_{\eta}^{a}-\frac{1}{\tilde{\theta}^{3}} \tilde{D}_{i} \tilde{F}_{i \eta}^{a}=0 .
\end{aligned}
$$

Substituting Eqs. (8), (9), and (10) into Eq. (7), we see that Gauss's law is expressed in terms of the conformal variables as

$$
\tilde{D}_{i}\left(\partial_{\theta} A_{i}^{a}-\frac{A_{i}^{a}}{2 \theta}\right)+\frac{1}{\tilde{\theta}^{3}} \tilde{D}_{\eta}\left(\partial_{\theta} A_{\eta}^{a}-\frac{A_{\eta}^{a}}{2 \theta}\right)=0 .
$$

\section{B. Background field configuration}

In this subsection, we introduce the gauge configuration which makes a homogeneous and boost-invariant longitudinal color magnetic field. In order to extract the essential ingredients of the non-Abelian gauge theory in a simple manner, we consider color SU(2). The color magnetic field in the $\tau-\eta$ coordinate is defined by
PHYSICAL REVIEW D 94, 016001 (2016)

$$
\mathcal{B}_{I}^{a}=\epsilon_{I J K}\left(\partial_{J} \mathcal{A}_{K}^{a}-\frac{1}{2} \epsilon^{a b c} \mathcal{A}_{J}^{b} \mathcal{A}_{K}^{c}\right) .
$$

From Eqs. (8) and (9), the color magnetic field in terms of the conformal variables are expressed as

$$
B_{I}^{a}=\epsilon_{I J K}\left(\tilde{\partial}_{J} A_{K}^{a}-\frac{1}{2} \epsilon^{a b c} A_{J}^{b} A_{K}^{c}\right)
$$

We assume that the gauge field itself is also homogeneous and boost invariant. One possible realization of the gauge configuration is given by

$$
\begin{gathered}
A_{i}^{a}=\tilde{A}(\theta)\left(\delta^{a 1} \delta_{i y}+\delta^{a 2} \delta_{i x}\right), \\
A_{\eta}^{a}=0,
\end{gathered}
$$

which fulfills the above requirements and exactly satisfies Gauss's law. Taking the configuration Eq. (21), only the second term in Eq. (20) remains. This is the generalization of the nonexpanding case $[29,30]$. We also mention that there exists not only color magnetic fields but also transversely polarized color electric fields.

The EOM of the background field is now given by

$$
\partial_{\theta}^{2} \tilde{A}+\frac{1}{4 \theta^{2}} \tilde{A}+\tilde{A}^{3}=0 .
$$

The second term comes from the quadratic term in the action which makes it difficult to get exact solutions analytically. Nonetheless, the qualitative behavior is well understood in some limiting cases as we shall show.

In the earlier time $\theta \ll 1 / \tilde{A}$, the self-interaction term $\tilde{A}^{3}$ can be neglected, and the EOM is reduced to a linear equation. Suppose that the initial condition is given as $\tilde{A}=\sqrt{B_{0}}$ and $\partial_{\theta} \tilde{A}=0$ at $\theta=\theta_{0}$; then, the solution is given by

$$
\tilde{A}(\theta) \simeq \sqrt{\frac{B_{0} \theta}{\theta_{0}}}\left(1-\frac{1}{2} \log \frac{\theta}{\theta_{0}}\right) .
$$

The nonlinear term dominates over the linear term in Eq. (23) after a time, and the EOM becomes nonlinear but solvable at the later times $\theta \gg 1 / \tilde{A}$ where the linear term is negligible. The background field should behave as

$$
\tilde{A}(\theta) \simeq B_{\mathrm{eff}}^{1 / 2} \operatorname{cn}\left(\theta B_{\mathrm{eff}}^{1 / 2}+\Delta ; 1 / \sqrt{2}\right),
$$

where $\operatorname{cn}(\theta ; k)$ is the Jacobi elliptic function of modulus $k$, which is a periodic function in time of which the period is $T=4 K(k) . K(k)$ is the complete elliptic integral of the first kind. Specifically, $T \simeq 7.42$ for $k=1 / \sqrt{2}$. This is nothing but the homogeneous background field discussed 
in Refs. [29,30] in the nonexpanding geometry with a phase shift $\Delta$ quantifying the earlier time effects.

In our setup, $B_{\text {eff }}^{1 / 2}$ is the only dimensionful scale, and its strength depends on the initial condition. It is convenient to introduce dimensionless quantities so as to normalize the final amplitude of the background gauge field in accordance with Eq. (25); that is, the final amplitude of $\tilde{A} / B_{\text {eff }}^{1 / 2}$ is normalized to unity, and the combination $\theta B_{\mathrm{eff}}^{1 / 2}$ is the dimensionless time variable.

Figure 1 shows the numerical solution of the EOM Eq. (23) rescaled by the final amplitude $B_{\text {eff }}^{1 / 2}$. We also show the solution of the linear equation Eq. (24), the Jacobi elliptic function, and the shifted Jacobi elliptic function Eq. (25). At the initial time, the initial gauge field and its derivative are chosen to be $\theta_{0}=0.01, \tilde{A}\left(\theta_{0}\right)=1$ and $d \tilde{A} / d \theta\left(\theta_{0}\right)=0$, respectively. The final amplitude and the time shift are found to be $B_{\text {eff }}^{1 / 2} \simeq 4.68$ and $\Delta \simeq 1.69$. Thus, the numerical result in Fig. 1 starts from $\theta_{0} B_{\mathrm{eff}}^{1 / 2} \simeq 0.01 \times 4.68$.

The linear solution Eq. (24) fails to reproduce the numerical solution at $\theta B_{\text {eff }}^{1 / 2} \gtrsim 1$ as expected. Let us compare this time with the transition time from the linear to the nonlinear regime $\theta_{\text {tr }}$ obtained from the balance condition $1 / \theta_{\text {tr }} \sim \tilde{A}\left(\theta_{\text {tr }}\right)$. Ignoring the logarithmic correction, we get $\theta_{\mathrm{tr}} \sim\left(\theta_{0} / B_{0}\right)^{1 / 3}$ from Eq. (24). By assuming $\theta_{\text {tr }} B_{\text {eff }}^{1 / 2} \sim 1$, we find $B_{\text {eff }} \sim\left(B_{0} / \theta_{0}\right)^{1 / 3}=(1 / 0.01)^{1 / 3} \simeq$ 4.64 for the present initial condition, which deviates from the numerical results only by around $1 \%$.

Before finishing this subsection, we comment on the physical time scale. From Eqs. (9), (11), (24), and (25), the solutions at the earlier and later times in the original $\tau-\eta$ coordinate are given by

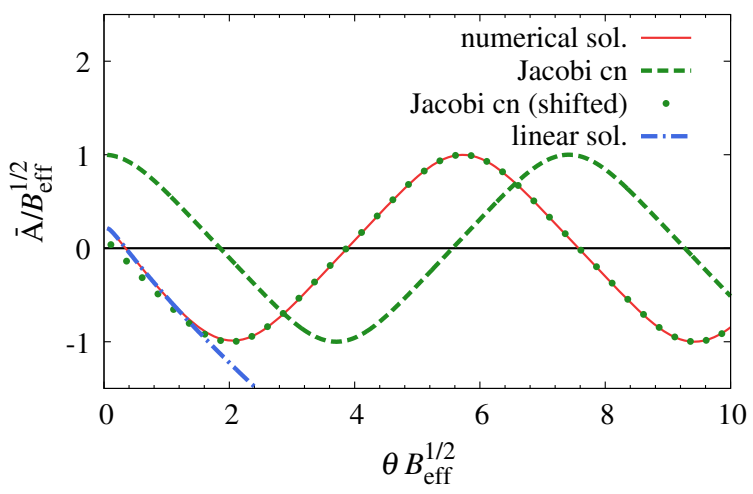

FIG. 1. Background field $\tilde{A}$ in an expanding geometry. We show the numerical solution of the EOM Eq. (23) rescaled by the asymptotic amplitude (red solid line), the linear solution Eq. (24) (blue dash-dotted line), the shifted Jacobi elliptic function Eq. (25) (green dotted line), and the Jacobi elliptic function (green dashed line). The temporal variable $\theta$ and the amplitude $\tilde{A}$ are normalized by the asymptotic amplitude $B_{\text {eff }}^{1 / 2}$.

$$
\begin{aligned}
\mathcal{A}_{y}^{1}(\tau) & =\mathcal{A}_{x}^{2}(\tau)=\tau^{-1 / 3} \tilde{A}(\theta) \\
& \simeq \begin{cases}\sqrt{\frac{3}{2}} B_{\mathrm{eff}}^{3 / 4}\left[1-\frac{1}{3} \log \left(\frac{\tau}{\tau_{0}}\right)\right] & \left(\theta B_{\mathrm{eff}}^{1 / 2} \ll 1\right), \\
\frac{B_{\text {eff }}^{1 / 2}}{\tau^{1 / 3}} \operatorname{cn}\left(\frac{3}{2} \tau^{2 / 3} B_{\mathrm{eff}}^{1 / 2}+\Delta ; \frac{1}{\sqrt{2}}\right) & \left(\theta B_{\mathrm{eff}}^{1 / 2} \gg 1\right),\end{cases}
\end{aligned}
$$

respectively. Equation (26) tells us the strength of the background gauge field. If the initial strength at $\tau_{0}=$ $\left(2 \theta_{0} / 3\right)^{3 / 2}$ is given by the saturation scale $Q_{\mathrm{s}}$, we get the relation between the physical and conformal scales,

$$
Q_{\mathrm{s}}=\sqrt{\frac{3}{2}} B_{\mathrm{eff}}^{3 / 4}, \quad Q_{\mathrm{s}} \tau=\frac{2}{3}\left(\theta B_{\mathrm{eff}}^{1 / 2}\right)^{3 / 2}
$$

For instance, we can evaluate the proper time as $Q_{\mathrm{s}} \tau=0.67,3.46$ and 21.1 for $\theta B_{\mathrm{eff}}^{1 / 2}=1,3$ and 10 , respectively.

\section{Equation of motion of fluctuations}

We write down the linearized EOM of fluctuations on top of the background gauge field Eq. (21). This is easily done by shifting $A_{I}^{a} \rightarrow A_{I}^{a}+a_{I}^{a}$ in Eqs. (16) and (17) and keeping terms of order $\mathcal{O}\left(a^{1}\right)$. The resultant EOM of fluctuations $a_{I}^{a}$ is given by

$\partial_{\theta}^{2} a_{i}^{a}+\frac{1}{9 \tilde{\theta}^{2}} a_{i}^{a}+\left[\Omega^{2}(\tilde{A})\right]_{i j}^{a b} a_{j}^{b}+\left[\Omega^{2}(\tilde{A})\right]_{i \eta}^{a b} a_{\eta}^{b}=0$,

$\frac{1}{\tilde{\theta}^{3}} \mathcal{L}_{\eta}^{2} a_{\eta}^{a}+\frac{7}{9 \tilde{\theta}^{5}} a_{\eta}^{a}+\left[\Omega^{2}(\tilde{A})\right]_{\eta j}^{a b} a_{j}^{b}+\left[\Omega^{2}(\tilde{A})\right]_{\eta \eta}^{a b} a_{\eta}^{b}=0$,

where $\mathcal{L}_{\eta}^{2}$ and $\Omega_{I}^{2}$ are defined by

$$
\mathcal{L}_{\eta}^{2}=\frac{d^{2}}{d \theta^{2}}-\frac{2}{\tilde{\theta}} \frac{d}{d \theta}
$$

and

$$
\begin{gathered}
{\left[\Omega^{2}\right]_{i j}^{a b}=-\left(\tilde{D}_{k}(\tilde{A}) \tilde{D}_{k}(\tilde{A})+\frac{1}{\tilde{\theta}^{3}} \tilde{D}_{\eta}(\tilde{A}) \tilde{D}_{\eta}(\tilde{A})\right)^{a b} \delta_{i j}} \\
+\left(\tilde{D}_{i}(\tilde{A}) \tilde{D}_{j}(\tilde{A})+2 i \tilde{F}_{i j}(\tilde{A})\right)^{a b} \\
{\left[\Omega^{2}\right]_{i \eta}^{a b}=\frac{1}{\tilde{\theta}^{3}}\left(\tilde{D}_{i}(\tilde{A}) \tilde{D}_{\eta}(\tilde{A})+2 i \tilde{F}_{i \eta}(\tilde{A})\right)^{a b}} \\
{\left[\Omega^{2}\right]_{\eta j}^{a b}=\frac{1}{\tilde{\theta}^{3}}\left(\tilde{D}_{\eta}(\tilde{A}) \tilde{D}_{j}(\tilde{A})+2 i \tilde{F}_{\eta j}(\tilde{A})\right)^{a b}} \\
{\left[\Omega^{2}\right]_{\eta j}^{a b}=-\frac{1}{\tilde{\theta}^{3}}\left(\tilde{D}_{k}(\tilde{A}) \tilde{D}_{k}(\tilde{A})\right)^{a b},}
\end{gathered}
$$


respectively. The color indices $a b$ appear in the covariant derivative $\tilde{D}$ and the field tensor $\tilde{F} . \Omega^{2}$ depends on conformal time not only explicitly but also implicitly through the background gauge field $\tilde{A}(\theta)$. Here and in the later discussions, we adopt the unit $B_{\mathrm{eff}}^{1 / 2}=1$. Namely, all variables are normalized by $B_{\text {eff }}^{1 / 2}$ such as $\theta B_{\text {eff }}^{1 / 2}$ and $a_{I}^{a} / B_{\mathrm{eff}}^{1 / 2}$.

Even though we consider the color magnetic background, both the transverse momenta $p_{i}$ and the longitudinal momentum $p_{\eta}$ are well defined because the background gauge field does not depend on spatial coordinates. Therefore, it is useful to introduce the Fourier representation of the EOM, Eqs. (28) and (29), through

$a_{I}^{a}(\theta, x, y, \eta)=\int \frac{d^{3} p}{(2 \pi)^{3}} a_{I}^{a}\left(\theta, p_{x}, p_{y}, p_{\eta}\right) e^{i\left(p_{x} x+p_{y} y+p_{\eta} \eta\right)}$.

It should be noted that we can set $p_{y}=0$ without loss of generality from rotational symmetry in the transverse plane. The matrix $\Omega^{2}$ at $p_{y}=0$ becomes block diagonal as $\Omega^{2}=\operatorname{diag}\left(\Omega_{4}^{2}, \Omega_{5}^{2}\right)$, as found in the nonexpanding case [30].

In the later discussion, we consider finite $p_{\eta}$ modes $\left(p_{\eta} \neq 0, p_{T}=0\right)$ and finite $p_{T}$ modes $\left(p_{\eta}=0, p_{T} \neq 0\right)$. From now on, $p_{T}$ denotes a transverse momentum. The EOMs of these modes at later times have the form of

$$
\frac{d^{2} a_{I}^{a}}{d \theta^{2}}+k_{\mathrm{eff}}^{2}(\theta) a_{I}^{a}+\lambda \mathrm{cn}^{2}(\theta+\Delta) a_{I}^{a}=R(\theta),
$$

and $d^{2} / d \theta^{2}$ is replaced with $\mathcal{L}_{\eta}^{2}$ for the longitudinal component $a_{\eta}^{a}$. Here, we defined the effective momenta $k_{\text {eff }}^{2}(\theta)$ for convenience. $R(\theta)$ denotes an inhomogeneous term. The effective momenta for the transverse and longitudinal components of the finite $p_{\eta}$ (finite $p_{T}$ ) modes, $k_{\text {eff }}^{2}=k_{\eta T}^{2}$ and $k_{\eta \eta}^{2}\left(k_{T T}^{2}\right.$ and $\left.k_{T \eta}^{2}\right)$, are defined by Eqs. (37)-(41) below. The explicit forms of the EOMs [namely, $R(\theta)$ ] are summarized in Appendix A.

For finite $p_{\eta}$ modes $\left(p_{T}=0\right)$, we find the effective momenta of the transverse and longitudinal components of fluctuations $a_{i}^{a}$ and $a_{\eta}^{a}$ given by

$$
\begin{gathered}
k_{\eta T}^{2}(\theta) \equiv M_{T}^{2}(\theta)+\frac{p_{\eta}^{2}}{\tilde{\theta}^{2}}=\frac{1+9 p_{\eta}^{2}}{4 \theta^{2}}, \\
k_{\eta \eta}^{2}(\theta) \equiv M_{\eta}^{2}(\theta)=\frac{7}{4 \theta^{2}}
\end{gathered}
$$

where $M_{T}^{2}$ and $M_{\eta}^{2}$ are effective masses defined by

$$
M_{T}^{2}(\theta)=\frac{1}{9 \tilde{\theta}^{2}}=\frac{1}{4 \theta^{2}}, \quad M_{\eta}^{2}(\theta)=\frac{7}{9 \tilde{\theta}^{2}}=\frac{7}{4 \theta^{2}} .
$$

Note that $k_{\eta T}^{2}$ and $k_{\eta \eta}^{2}$ decrease in time monotonically.

In the same manner, we define the effective momenta for finite $p_{T}$ modes $\left(p_{\eta}=0\right)$ by

$$
\begin{aligned}
& k_{T T}^{2}(\theta) \equiv M_{T}^{2}(\theta)+\tilde{\theta} p_{T}^{2}=\frac{1}{4 \theta^{2}}+\frac{2}{3} \theta p_{T}^{2}, \\
& k_{T \eta}^{2}(\theta) \equiv M_{\eta}^{2}(\theta)+\tilde{\theta} p_{T}^{2}=\frac{7}{4 \theta^{2}}+\frac{2}{3} \theta p_{T}^{2} .
\end{aligned}
$$

In contrast to the finite $p_{\eta}$ modes Eqs. (37) and (38), $k_{T T}^{2}$ and $k_{T \eta}^{2}$ increase at later times.

Before we close this section, we comment on the similarities and differences in EOMs in the expanding and nonexpanding geometries. The forms of EOMs expressed by using the conformal variables Eqs. (28) and (29) are similar to those in a nonexpanding geometry. For example, the background field at later times is described by the elliptic function; then, the Eq. (36) is similar to Lamé's equation, which appears in the nonexpanding geometries and discussed in the next section. In addition, $\Omega^{2}$ is block diagonalized in the same way as in the nonexpanding case [30]. On the other hand, the form of EOMs in the expanding geometry is different from that in the nonexpanding geometry in the appearance of the time dependence of the effective transverse and longitudinal momenta. Thus, the original problem defined in the expanding geometry is mapped into the nonexpanding problem where the momenta of the fluctuations depend on time.

\section{FLOQUET THEORY}

In general, a temporally periodic-driven system can show instability due to the resonance between an external force and eigenmodes of the system. The resultant instability is called parametric resonance or parametric instability. It is well known that the Floquet theory is best suited to analyze instabilities of temporally periodic-driven systems. Floquet theory provides the criteria whether the solution is exponentially divergent, polynomially divergent, or bounded. In the expanding problem, Floquet theory is not directly applied but gives some insights into the growth rates in the expanding geometry. An account of the Floquet theory is given in our previous paper [30]. In this section, we recapitulate them to be self-contained in a brief way.

Let us consider Lamé's equation

$$
\ddot{f}+\left[p^{2}+\lambda \mathrm{cn} n^{2}(t ; k)\right] f=0 .
$$

Here, $p$ and $\lambda$ are control parameters of this equation. It should be noted that, at later times, EOMs in the expanding geometry, Eq. (36), become the Lamé equation if we ignore the time dependence of the effective momenta and the 
inhomogeneous terms. In the context of the CYM theory, the cases with $p \ll 1$ and $\lambda= \pm 1,2,3$ are important. Let us define a fundamental matrix by $\Phi(t)=\left(\left(f_{1}, \dot{f}_{1}\right)^{t},\left(f_{2}, \dot{f}_{2}\right)^{t}\right)$, where $\left\{f_{i}\right\}_{(i=1,2)}$ are independent and complete solutions of the Lamé equation. If $\Phi(t)$ is a fundamental matrix, or in this specific case a Wronskian matrix, $\Phi(t+T)$ is also a fundamental matrix due to the periodicity of the elliptic function, where $T$ is the period of the elliptic function.

The criterion of the existence of unstable solutions is expressed in terms of the monodromy matrix $M$ defined by $\Phi(t+T)=\Phi(t) M$ which is regular and time independent. By construction, the fundamental matrix is also regular, and we get

$$
M=\Phi(0)^{-1} \Phi(T) .
$$

Because the Wronskian det $\Phi$ is constant in time, we also find $\operatorname{det} M=\mu_{1} \mu_{2}=1$. The eigenvalues of $M$ are called characteristic multipliers, and we denote them as $\mu_{1}$ and $\mu_{2}$. These multipliers are the solutions of the characteristic equation: $\mu^{2}-(\operatorname{tr} M) \mu+1=0$.

One can easily show that the solution of the Lamé equation must have the following form,

$$
\Phi(t)=F(t) \exp \left[(\log M) \frac{t}{T}\right]
$$

where $F(t)$ is a $T$-periodic matrix of which the specific form is irrelevant to our discussion. It is useful to define the characteristic exponent or growth rate by $\gamma=(\log \mu) / T$. Thus, the eigenvalues of the monodromy matrix determine the stability of the solution. The complete classification is listed below:

(1) If $|\operatorname{tr} M|>2$, the solution is exponentially divergent.

(2) If $|\operatorname{tr} M|=2$, the solution is (anti)periodic or linearly divergent.

(3) If $|\operatorname{tr} M|<2$, the solution is bounded.

For more complete discussions about linear divergence, see also Appendix B. Figure 2 shows that the real part of the

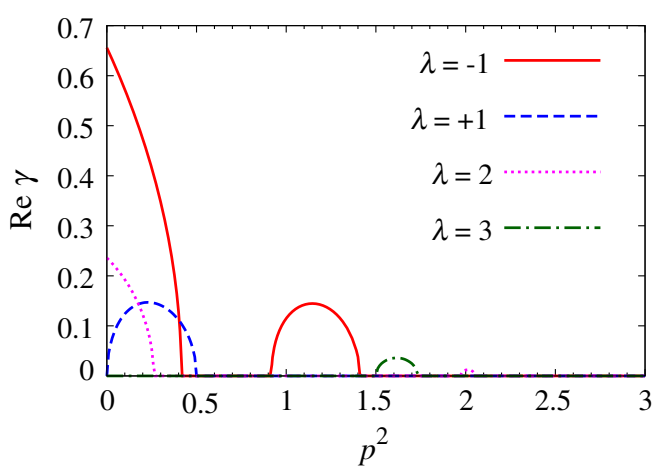

FIG. 2. Growth rates in Lamé's equation. We show the real part of the growth rates for $\lambda=-1$ (red solid line), $\lambda=1$ (blue dashed line), $\lambda=2$ (magenta dotted line), and $\lambda=3$ (green dotdashed line). growth rates of Lamé's equation for $\lambda= \pm 1,2$ and 3 . The growth rate has a maximum at $p^{2}=0$ for $\lambda=-1$ and 2 , the values of which are 0.66 and 0.23 , respectively. For $\lambda=1$ and 3 , the exponential growth rates are equal to zero at $p=0$, and the solutions are bounded or diverge at most linearly [30].

\section{STABILITY ANALYSIS}

In this section, we perform a linear stability analysis for fluctuations by solving the EOMs, Eqs. (28) and (29), around the background field (21). We first show the numerical results in Sec. IVA, and then in the following subsections, we shall give a semianalytical analysis in some limits, which helps to understand the numerical results. We shall only show the results for finite $p_{\eta}$ modes $\left(p_{\eta} \neq 0\right.$, $\left.p_{T}=0\right)$ and finite $p_{T}$ modes $\left(p_{\eta}=0, p_{T} \neq 0\right)$, which should be sufficient to see how instabilities emerge in an expanding system. The details including all explicit expressions of the equations are presented in Appendix A.

\section{A. Numerical results}

We choose the initial conditions for fluctuations so that Gauss's law Eq. (18) is satisfied, which leads to

$$
\begin{aligned}
& i \tilde{p}_{i} \dot{a}_{i}^{a}+\tilde{\theta}_{0}^{-3} i \tilde{p}_{\eta} \dot{a}_{\eta}^{a}-\frac{1}{3 \tilde{\theta}_{0}}\left(i \tilde{p}_{i} a_{i}^{a}+\tilde{\theta}_{0}^{-3} i \tilde{p}_{\eta} a_{\eta}^{a}\right) \\
& \quad+\epsilon^{a b c} \delta^{b 2}\left(\tilde{A}\left(\theta_{0}\right) \dot{a}_{x}^{c}-\dot{\tilde{A}}\left(\theta_{0}\right) a_{x}^{c}\right) \\
& \quad+\epsilon^{a b c} \delta^{b 1}\left(\tilde{A}\left(\theta_{0}\right) \dot{a}_{y}^{c}-\dot{\tilde{A}}\left(\theta_{0}\right) a_{y}^{c}\right)=0,
\end{aligned}
$$

where $\tilde{p}_{I}=\tilde{\theta}_{0}^{1 / 2} p_{I}$. We can choose the initial transverse components as $a_{i}^{a}=1$ and $\partial_{\theta} a_{i}^{a}=0$ in the rescaled dimensionless unit [36]. The initial longitudinal components are determined by Gauss's law. The initial condition of the background field is the same as that shown in Sec. II B; $\theta_{0} B_{\text {eff }}^{1 / 2} \simeq 0.01 \times 4.68, \tilde{A} / B_{\text {eff }}^{1 / 2} \simeq 1 / 4.68$, and $\partial_{\theta} \tilde{A} / B_{\text {eff }}^{1 / 2}=0$ in the rescaled dimensionless unit.

Figures 3 and 4 show the numerical results of squared fluctuation amplitudes obtained by solving the EOM given by Eq. (29) for finite $p_{\eta}$ and finite $p_{T}$ modes, respectively. For comparison, we show the exponential functions $e^{2 \gamma_{0} \theta}$ by black solid lines in those modes where exponential growth is expected. The comparison shows that there are several exponentially unstable modes. The amplitudes of $a_{A+}$ and $a_{B-}$ components in finite $p_{\eta}$ modes obey the Lamé equation with $\lambda=-1$ at later times and have a growth rate $\gamma_{0} \simeq 0.66$. The Lamé equation with $\lambda=-1$ describes the most unstable mode also in the nonexpanding geometry. Therefore, we conclude that the parametric instability emerges also in the expanding geometry for finite $p_{\eta}$ modes. Finite $p_{T}$ modes also show instability when $p_{T}$ is small but finite. 
PARAMETRIC INSTABILITY OF CLASSICAL YANG- ...
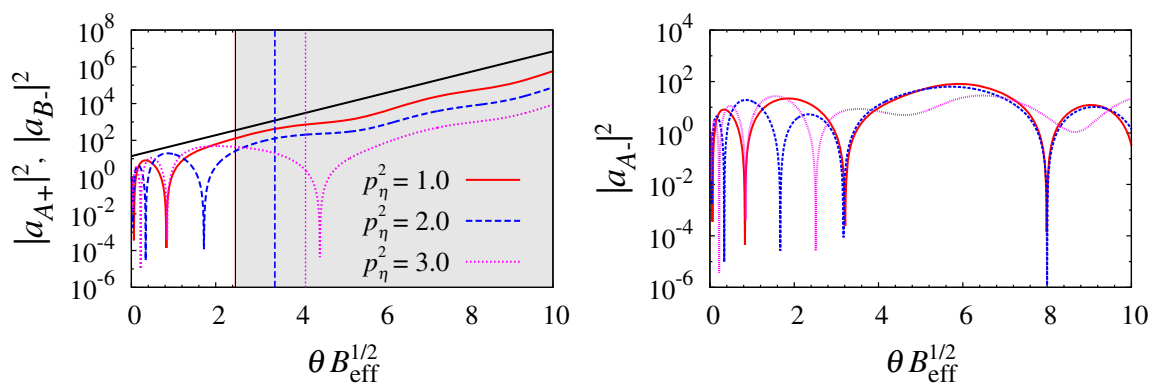

PHYSICAL REVIEW D 94, 016001 (2016)
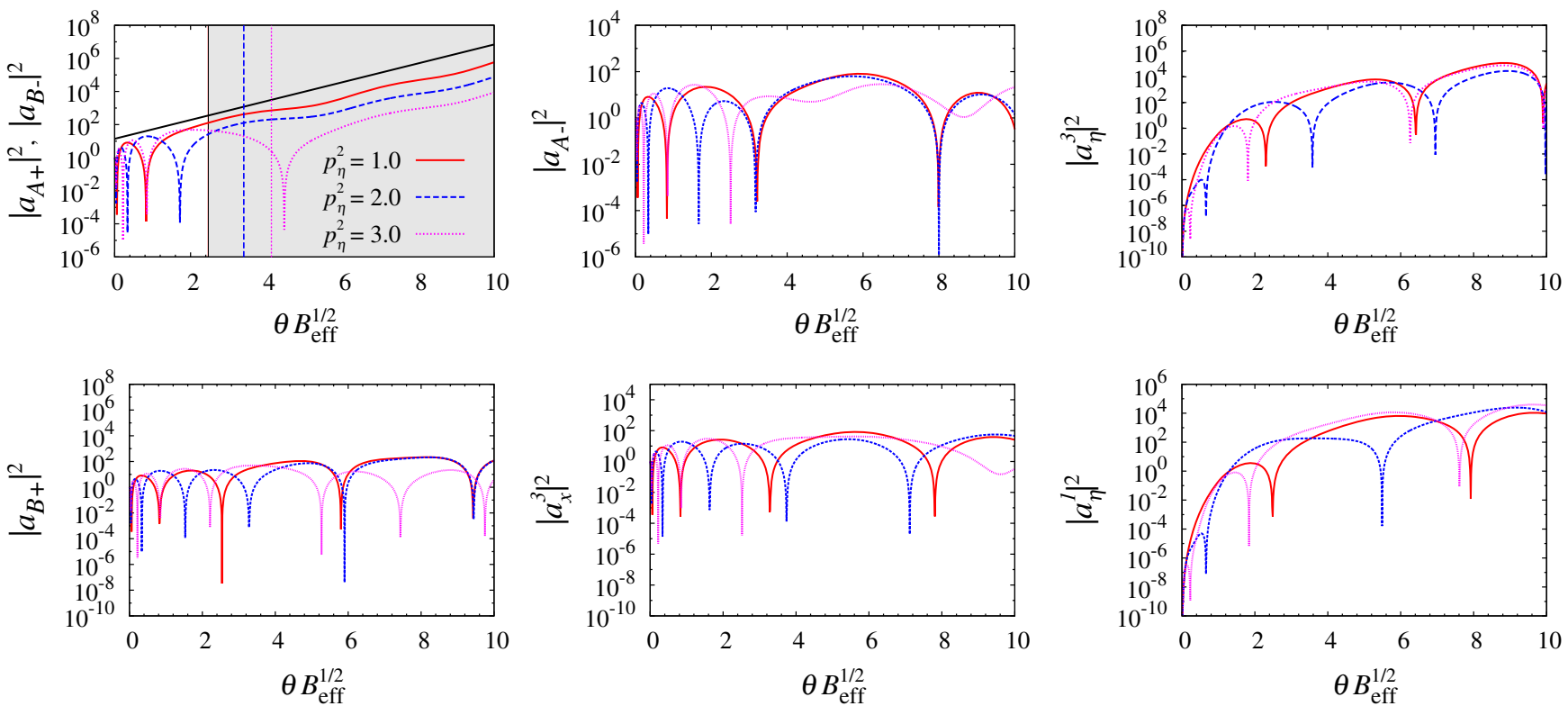

FIG. 3. Squared fluctuation amplitudes in finite $p_{\eta}$ modes. We show the time evolution of fluctuations in finite $p_{\eta}$ modes for $p_{\eta}^{2}=1.0,2.0$, and 3.0. Black solid lines in the upper panels show exponential function $e^{2 \gamma_{0} \theta}$ with $\gamma_{0}=0.66$. The vertical lines show the transition times for each momentum mode. The transition times of $a_{A+}, a_{B-}$ are given by $2.46,3.40$, and 4.13 for each momentum mode. The transition time of $a_{\eta}^{3}$ is 1.97 and independent of $p_{\eta}$. Shaded areas show the time regime where exponential growth is expected for $p_{\eta}^{2}=1.0$.

We also find that unstable modes grow exponentially after some times or in a limited time regime and show oscillating behavior outside of the exponential regime. The transitions between the oscillating behavior and the exponential growth occur at certain times marked by vertical lines in Figs. 3 and 4. For typical momenta, say $p_{\eta}^{2}=1.0$, the exponential growth is observed in the shaded areas while fluctuation amplitudes just oscillate outside the areas. The oscillatory behavior in the earliest stage is caused by the nonperiodic evolution of the background field.

In the following subsections, we interpret these numerical results in detail, namely, clarifying the difference between finite $p_{\eta}$ modes and $p_{T}$ modes. In Sec. IV B, we discuss the evolution in the earliest stage. In Secs. IV C and IV D, we estimate the growth rates and the transition times of the unstable modes. In Sec. IV E, we discuss the applicability range of the linear stability analysis. Finally, in Sec. IV F, we comment on the physical time scale of the parametric instability and the relevance to the heavy-ion experiments.

\section{B. Earliest stage}

We discuss the behavior of fluctuations in the earliest stage characterized by $\theta \ll 1$. In this stage, the effective momenta give rise to the most singular terms $k_{\text {eff }}^{2} \propto \theta^{-2}$, which is much larger than the background field $\tilde{A} \sim \theta^{1 / 2}$ as given in Eq. (24).
The EOMs for finite $p_{\eta}$ modes in the earliest stage read

$$
\begin{aligned}
\ddot{a}_{i}^{a}+k_{\eta T}^{2}\left(\theta, p_{\eta}\right) a_{i}^{a} & =f(\theta), \\
\mathcal{L}_{\eta}^{2} a_{\eta}^{a}+M_{\eta}^{2}(\theta) a_{\eta}^{a} & =f(\theta) .
\end{aligned}
$$

Here, $f(\theta)$ denotes higher order terms in $\theta$ which are irrelevant in this stage. Both of the effective momenta, $k_{\eta T}^{2}$ and $k_{\eta \eta}^{2}=M_{\eta}^{2}$ given in Eqs. (37) and (38), are proportional to $\theta^{-2}$. The general solutions for the transverse components are given by $a_{i}^{a}=c \theta^{1 / 2+3 i p_{\eta} / 2}+$ c.c. where $c$ is an arbitrary constant; one sees that the $p_{\eta}$ dependence of $k_{\eta T}^{2}$ causes the oscillating behavior of the solution. The longitudinal components do not have $p_{\eta}$ dependence, and the general solutions are given by $a_{\eta}^{a}=c_{1} \theta^{1 / 2}+c_{2} \theta^{7 / 2}$.

For finite $p_{T}$ modes, we have

$$
\begin{aligned}
& \partial_{\theta}^{2} a_{i}^{a}+M_{T}^{2}(\theta) a_{i}^{a}=\mathcal{O}\left(\theta^{-2 / 3}, \theta\right), \\
& \mathcal{L}_{\eta}^{2} a_{\eta}^{a}+M_{\eta}^{2}(\theta) a_{\eta}^{a}=\mathcal{O}\left(\theta^{-2 / 3}, \theta\right),
\end{aligned}
$$

where $\mathcal{O}\left(\theta^{-2 / 3}, \theta\right)$ indicates that the term is of order $\mathcal{O}\left(\theta^{-2 / 3}\right)$ or $\mathcal{O}(\theta)$. In the earliest stage, $p_{T}$ dependence appears in $\mathcal{O}(\theta)$ terms, as found in Eqs. (40) and (41). Note that EOM of the transverse components has the same form as that of the background field; then, the general solution is given by $a_{i}^{a}=c_{1} \theta^{1 / 2}+c_{2} \theta^{1 / 2} \log \theta$. The solution for the 
TSUTSUI, KUNIHIRO, and OHNISHI
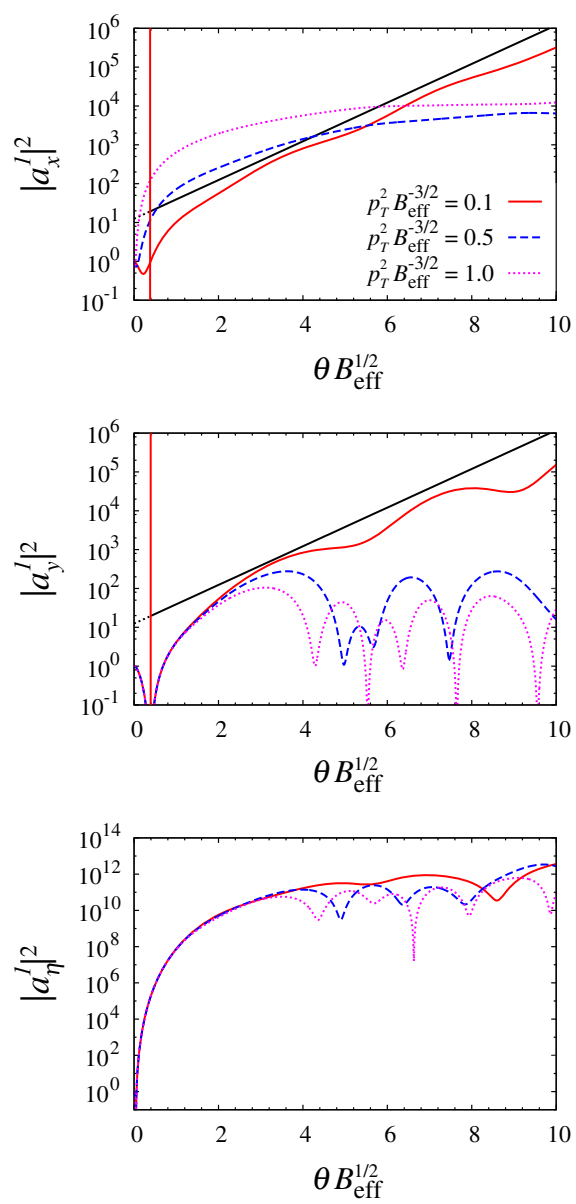
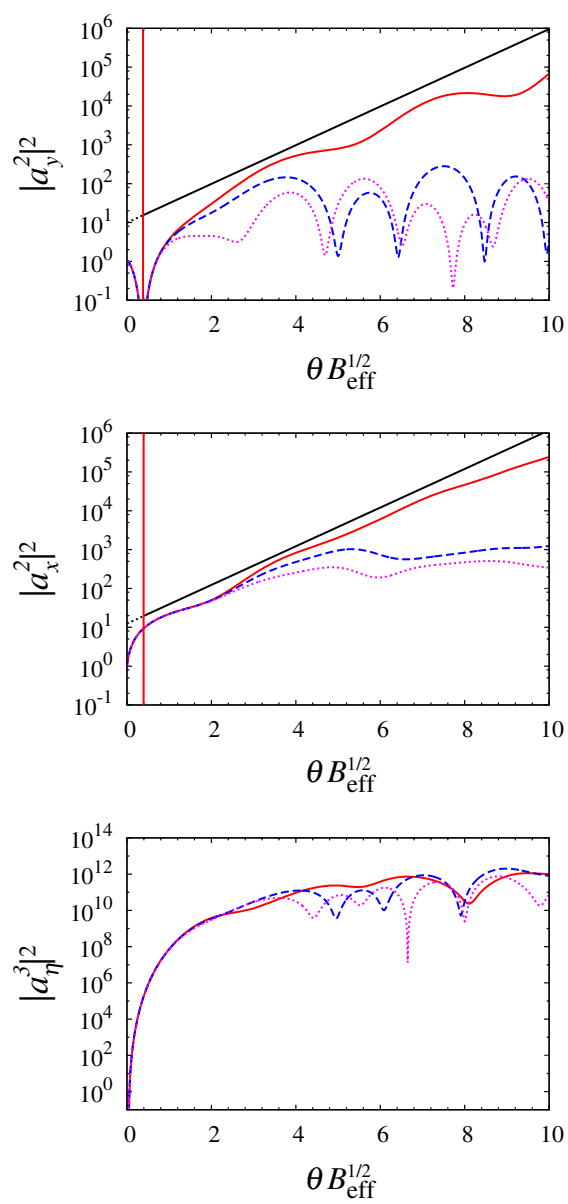

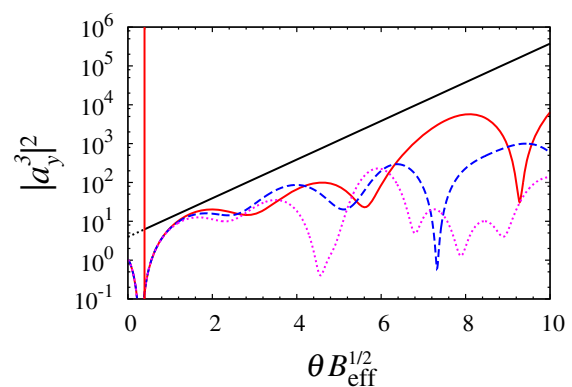

PHYSICAL REVIEW D 94, 016001 (2016)
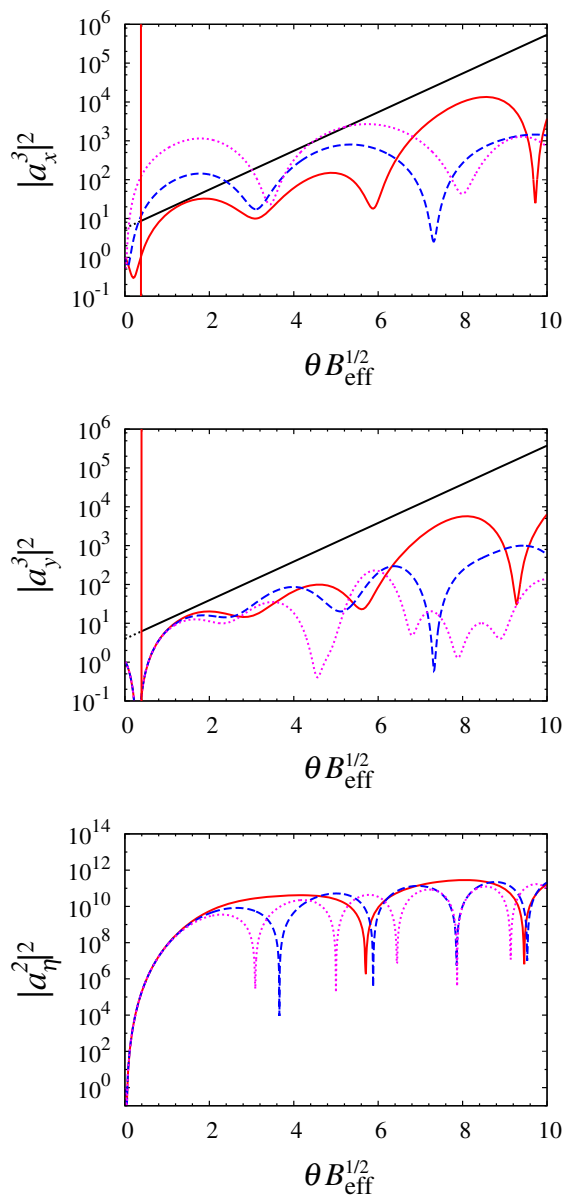

FIG. 4. Squared fluctuation amplitudes in finite $p_{T}$ modes. We show the time evolution of fluctuations in finite $p_{T}$ modes for $p_{T}^{2}=0.1,0.5$, and 1.0. In the upper and middle panels, the growth rates of the function $e^{2 \tilde{\gamma} \theta}$ are given by $\tilde{\gamma}=0.57$ for $p_{T}^{2}=0.1$. Two transition times are indicated with vertical lines for each momentum mode. In the $\Omega_{5}^{2}$ sector which includes $a_{x}^{1}, a_{y}^{2}, a_{x}^{3}$, the first transition time is given by $0.38,0.39$, and 0.41 for $p_{T}^{2}=0.1,0.5$, and 1.0 , respectively. In the $\Omega_{4}^{2}$ sector which includes $a_{y}^{1}$, $a_{x}^{2}$, $a_{y}^{3}$, the first transition time is given by 0.39 and 0.41 for $p_{T}^{2}=0.1$ and 0.5 , respectively.

longitudinal components grows in time with the same time dependence as the solution of finite $p_{\eta}$ modes.

From the above arguments, we find that fluctuation amplitudes in both finite $p_{\eta}$ and $p_{T}$ modes behave as $a \propto \theta^{1 / 2}+$ (higher order of $\theta$ ) in the earliest stage, except for the logarithmic corrections in $\theta$. The fluctuation amplitude in the $\tau-\eta$ coordinate is given by $\tau^{-1 / 3} a$ from Eqs. (9) and (10); then, they will not grow as a function of the proper time, i.e. $\tau^{-1 / 3} a(\tau) \propto \tau^{-1 / 3} \theta(\tau)^{1 / 2}=\mathcal{O}\left(\tau^{0}\right)$, if we ignore logarithmic corrections. The numerical results are in good agreement with the above discussion. In fact, no exponential growth is found in Figs. 3 and 4 at the earliest stage.

\section{Growth rates}

We shall now discuss exponential instability of fluctuations. In Figs. 3 and 4, there are some exponentially growing modes, namely, $a_{A_{+}}$and $a_{B_{-}}$components of the finite $p_{\eta}$ modes and $a_{x}^{a}$ and $a_{y}^{a}$ components with $p_{T}^{2}=0.1$.
As we shall see below, the growth rates of them are nicely described by utilizing the Floquet analysis in the nonexpanding geometry which was worked out in the previous work [30]: the (time-dependent) effective momenta in the expanding geometry are mapped in the contour map of the instability bands for the nonexpanding geometry. In the following analysis, we shall first recapitulate and utilize the results in the previous work for later convenience.

In Fig. 5, we show the maximal growth rate in the nonexpanding geometry as a function of transverse and longitudinal momenta. The contour maps are obtained for $\Omega_{4}^{2}$ and $\Omega_{5}^{2}$ sectors, separately. Figure 5 clearly shows the band structure of the parametric instability. The boundaries of instability bands are depicted by gray solid lines. We note that the zero momentum mode is most unstable, and its growth rate is given by

$$
\gamma_{0} \simeq 0.66
$$




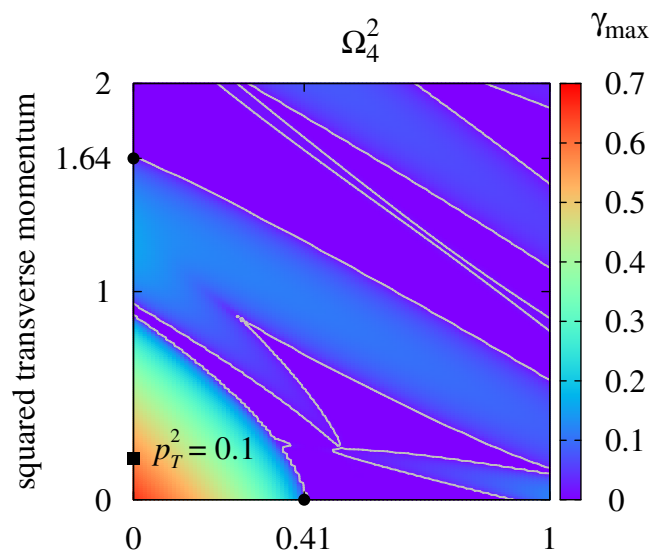

squared longitudinal momentum

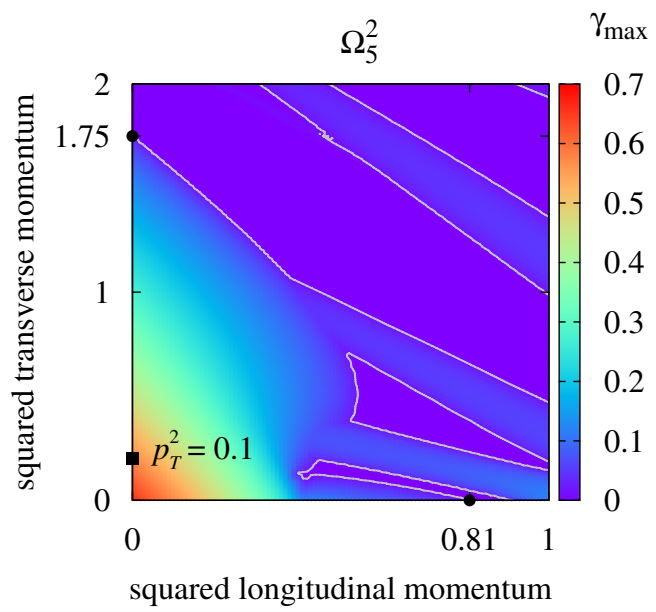

FIG. 5. Maximal growth rates as functions of transverse and longitudinal momenta for $\Omega_{4}^{2}$ sector (top) and $\Omega_{5}^{2}$ sector (bottom) in the nonexpanding geometry. The band boundaries denoted by gray solid lines are determined by $|\gamma|_{\max }=0.01$. Black squares denote the minimum values of $k_{T T}$. In both sectors, min $k_{T T}=$ 0.20 and $\gamma=0.57$ for $p_{T}^{2}=0.1$.

These results in the nonexpanding geometry enable us to estimate the growth rates of the numerical solutions in the expanding geometry by paying attention to the time dependence of the effective momenta.

Let us evaluate the growth rates in the finite $p_{\eta}$ modes by using the growth rates in the nonexpanding geometry. We neglect terms with negative powers of $\theta$ and obtain the asymptotic EOMs as

$$
\frac{d^{2} a_{I}^{a}}{d \theta^{2}}+\lambda \mathrm{cn}^{2}(\theta+\Delta) a_{I}^{a}=R(\theta)
$$

where $\lambda= \pm 1,2$, or 3 , and we have replaced the background field with its asymptotic form, $\tilde{A} \sim \operatorname{cn}(\theta+\Delta ; 1 / \sqrt{2})$ shown in Eq. (25). These equations are nothing but Lamé's equation with inhomogeneous terms $R(\theta)$. [See Eqs. (A5)-(A12) for full expression of $R(\theta)$ and details of the derivation.] The solutions are unstable for $\lambda=-1$ and 2 due to the parametric instability as we show in Fig. 2. Their growth rates are given by those of the zero momentum mode in the nonexpanding geometry and are found to be $\gamma=0.66$ and $\gamma=0.23$ for $\lambda=-1$ and $\lambda=2$, respectively. We summarize instability properties of finite $p_{\eta}$ modes in Table I.

For the finite $p_{T}$ modes, we need to be cautious about the nonmonotonic dependence of the effective momenta on $\theta$. The EOMs of the finite $p_{T}$ modes are given in Eqs. (A15)-(A23). The effective transverse momentum Eq. (40) first decreases, takes a minimum at a finite positive value, and increases again with increasing $\theta$. We expect that the maximum growth rate may be evaluated at which $k_{T T}$ has the minimum value. In both $\Omega_{5}^{2}$ and $\Omega_{4}^{2}$ sectors, the growth rate at the minima of $k_{T T}$, marked by squares in Fig. 5, reads

$$
\tilde{\gamma} \simeq 0.57
$$

for $p_{T}^{2}=0.1$.

We now examine the growth rates estimated from the Floquet analysis by comparing them with the numerical results in the expanding geometry. In the upper panels of Fig. 3, we compare the numerical results of finite $p_{\eta}$ modes with exponential functions $\exp \left(2 \gamma_{0} \theta\right)$ shown by the solid line, where $\gamma_{0}$ is the growth rate of the zero momentum mode obtained in the nonexpanding geometry, Eq. (50). The numerical solutions are plotted for $p_{\eta}^{2}=1.0,2.0$, and 3.0. The unstable components are $a_{A+}=\left(a_{x}^{1}+a_{y}^{2}\right) / \sqrt{2}$ and $a_{B-}=\left(a_{y}^{1}-a_{x}^{2}\right) / \sqrt{2}$ of which the asymptotic growth rate is expected to be $\gamma=0.66$ from the (inhomogeneous) Lamé equation Eq. (51) with $\lambda=-1$. As expected, the growth rates for $a_{A+}$ and $a_{B-}$ in the expanding geometry approach the upper bound determined by those in a nonexpanding geometry. We have found that other components such as $a_{A-}^{3}$ and $a_{\eta}^{3}$ exhibit only oscillating

TABLE I. The classification of the asymptotic behavior for finite $p_{\eta}\left(p_{T}=0\right)$ modes. Here, $a_{A \pm}=\left(a_{x}^{1} \pm a_{y}^{2}\right) / \sqrt{2}$ and $a_{B \pm}=\left(a_{y}^{1} \pm a_{x}^{2}\right) / \sqrt{2}$. * For the $a_{A-}$ component at $p_{\eta}=0$, the inhomogeneous term $R(\theta)$ vanishes and shows only linear divergence as ordinal instability of Lamé's equation with $\lambda=1$.

\begin{tabular}{lccccc}
\hline \hline Sector Component & $\lambda$ & $R(\theta)$ & Instability & Growth rate \\
\hline$\Omega_{5}^{2}$ & $a_{A+}$ & -1 & $R(\theta) \neq 0$ & Exponential & $\gamma=0.66$ \\
$\Omega_{5}^{2}$ & $a_{A-}$ & +1 & $(*) R(\theta) \neq 0$ & Exponential* & $\gamma=0.23$ \\
$\Omega_{4}^{2}$ & $a_{B+}$ & +3 & $R(\theta)=0$ & Linear & $\ldots$ \\
$\Omega_{4}^{2}$ & $a_{B-}$ & -1 & $R(\theta)=0$ & Exponential & $\gamma=0.66$ \\
$\Omega_{5}^{2}$ & $a_{x}^{3}$ & +1 & $R(\theta)=0$ & Linear & $\ldots$ \\
$\Omega_{4}^{2}$ & $a_{y}^{3}$ & +1 & $R(\theta)=0$ & Linear & $\ldots$ \\
$\Omega_{5}^{2}$ & $a_{\eta}^{1}$ & +1 & $R(\theta) \neq 0$ & Linear & $\ldots$ \\
$\Omega_{4}^{2}$ & $a_{\eta}^{2}$ & +1 & $R(\theta) \neq 0$ & Linear & $\ldots$ \\
$\Omega_{5}^{2}$ & $a_{\eta}^{3}$ & +2 & $R(\theta) \neq 0$ & Exponential & $\gamma=0.23$ \\
\hline \hline
\end{tabular}


TSUTSUI, KUNIHIRO, and OHNISHI

behavior within the time range shown in Fig. 3, although, according to Table I, $a_{A-}^{3}$ and $a_{\eta}^{3}$ can be unstable. This is due to the fact that they have too small a growth rate. In principle, one will find the exponential growth of these components by carrying out unreasonably long-time simulation, where the linear analysis is no longer valid.

Let us turn to the finite $p_{T}$ modes. In Fig. 4 , we compare the exponential functions $\exp (2 \tilde{\gamma} \theta)$ with numerical solutions. Here, $a_{y}^{1}, a_{x}^{2}$, and $a_{y}^{3}$ belong to the $\Omega_{4}^{2}$ sector, and $a_{x}^{1}$, $a_{y}^{2}$, and $a_{x}^{3}$ belong to the $\Omega_{5}^{2}$ sector. At earlier times, the growth rate Eq. (52) agrees well with that of the numerical solutions for $p_{T}^{2}=0.1$. As the effective momenta become large, the numerical solutions deviate from the exponential functions $\exp (2 \tilde{\gamma} \theta)$. This behavior is in accordance with the fact that there is no significant instability band in the high transverse momentum region.

\section{Transition times}

We now discuss the temporal regime where the exponential growth is expected. The main difference between nonexpanding and expanding geometries comes from the time dependence of the effective momenta $k_{\text {eff }}^{2}=k_{\eta T}^{2}, k_{\eta \eta}^{2}$, $k_{T T}^{2}$, or $k_{T \eta}^{2}$, defined in Eqs. (37), (38), (40), and (41). They control the transition between oscillating behavior and the exponential growth; when $k_{\mathrm{eff}}^{2}$ is in the instability bands of the corresponding nonexpanding case, we can expect exponential growth. The shaded area in the top left panel of Fig. 3 shows the temporal regime, where exponential growth is expected.

Figure 6 shows the time dependence of the effective momenta $k_{\eta T}^{2}, k_{\eta \eta}^{2}$, and $k_{T T}^{2}$. The horizontal lines correspond to the boundaries of the instability bands. For finite $p_{\eta}$ modes, the effective momenta (37) and (38) decrease in time, and then the infrared band structure of the nonexpanding geometry is relevant and responsible for the emergence of instabilities. Two horizontal lines in the top panel of Fig. 6 represent the longitudinal momenta on the boundaries of the instability band around the zero momentum region, 0.41 for the $\Omega_{4}^{2}$ sector and 0.81 for the $\Omega_{5}^{2}$ sector. Using the momentum on the boundary point $p_{b \eta}$, we define the transition time $\theta_{\text {tr }}$ by

$$
\begin{gathered}
p_{b \eta}^{2}=k_{\eta T}^{2}\left(\theta_{\mathrm{tr}}\right)=M_{T}^{2}\left(\theta_{\mathrm{tr}}\right)+9 p_{\eta}^{2} / 4 \theta_{\mathrm{tr}}^{2}, \\
p_{b \eta}^{2}=k_{\eta \eta}^{2}\left(\theta_{\mathrm{tr}}\right)=M_{\eta}^{2}\left(\theta_{\mathrm{tr}}\right)
\end{gathered}
$$

for transverse and longitudinal components, respectively. Note that the latter has no $p_{\eta}$ dependence. Specifically, the transition time of the most unstable modes $a_{A+}(p=0)$ and $a_{B-}(p=0)$ reads $p_{b \eta}^{2}=M_{T}^{2}\left(\theta_{\text {tr }}\right)$. Then, we obtain

$$
\theta_{\mathrm{tr}}\left(p_{\eta}=0\right) \simeq 0.78
$$

PHYSICAL REVIEW D 94, 016001 (2016)
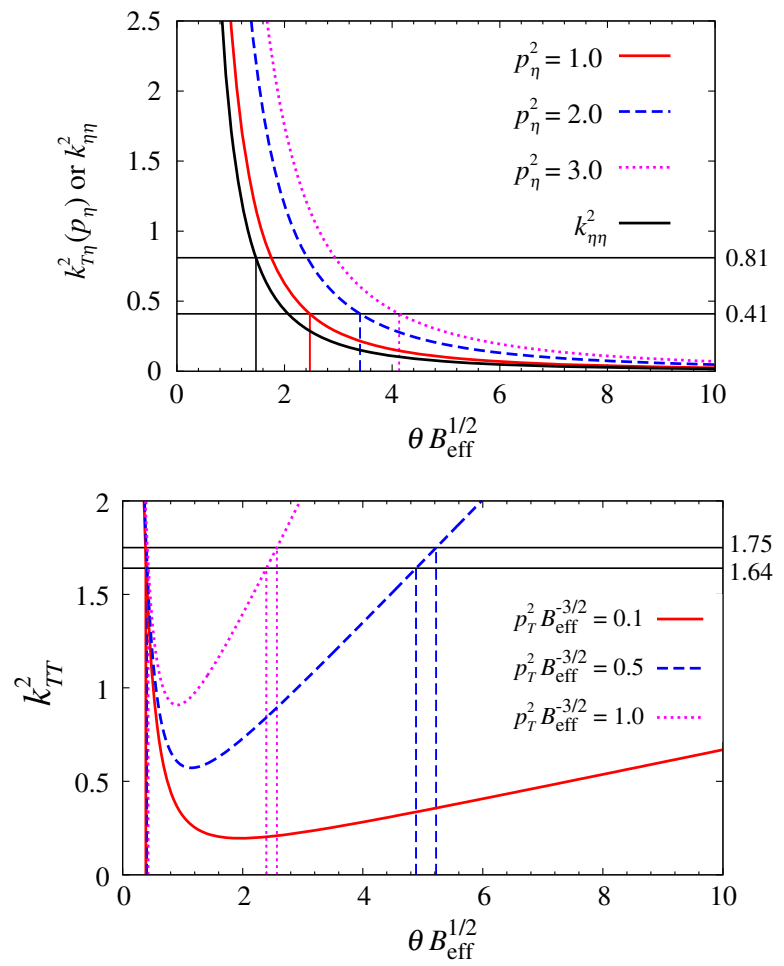

FIG. 6. Time dependence of the effective momenta. The horizontal lines show the boundaries of the instability bands. (Upper panel) Effective momenta in finite $p_{\eta}$ modes. The boundaries are given by $k_{T \eta}^{2}\left(\right.$ or $\left.k_{\eta \eta}^{2}\right)=p_{b \eta}^{2}=0.81$ and $k_{T \eta}^{2}\left(\right.$ or $\left.k_{\eta \eta}^{2}\right)=0.41$. (Lower panel) Effective momenta of finite $p_{T}$ modes. The boundaries are given by $k_{T T}^{2}=p_{b T}^{2}=1.75$ and $k_{T T}^{2}=1.64$. In both panels, vertical lines show the transition times.

with $p_{b \eta}^{2}=0.41$. The transition times at $p_{\eta}^{2}=1.0$ are $\theta_{\mathrm{tr}}=2.46(1.46)$ for $a_{A+}$ and $a_{B-}\left(a_{\eta}^{3}\right)$ components. The transition times defined here are denoted by vertical lines in Fig. 3.

For finite $p_{T}$ modes, there is a finite time interval in which the effective momenta stay inside of the instability bands. Two horizontal lines in the lower panel of Fig. 6 represent the transverse momenta on the band boundaries, 1.64 for the $\Omega_{4}^{2}$ sector and 1.75 for the $\Omega_{5}^{2}$ sector. All unstable components are transverse $a_{i}^{a}$, so the transition time $\theta_{\text {tr }}$ is defined by

$$
p_{b T}^{2}=k_{T T}^{2}\left(\theta_{\mathrm{tr}}\right)=M_{T}^{2}\left(\theta_{\mathrm{tr}}\right)+\frac{2}{3} \theta_{\mathrm{tr}} p_{T}^{2} .
$$

Since effective momenta are not monotonic in time, there are two transition times. For instance, the first transition time at $p_{T}^{2}=0.1$ is $\theta_{\mathrm{tr}}=0.39(0.38)$ for the $\Omega_{4}^{2}\left(\Omega_{5}^{2}\right)$ sector, which is the starting time of the exponential growth. The second transition time is $\theta_{\text {tr }}=24.6(26.2)$ for the $\Omega_{4}^{2}\left(\Omega_{5}^{2}\right)$ sector, which is the finishing time of the exponential growth. Thus, this mode has a finite time interval in which 
an exponential growth is expected for small $p_{T}$. Indeed, unstable behavior is clearly observed in Fig. 4 at $p_{T}^{2}=0.1$.

We discuss the behavior of high transverse momentum modes. In contrast to the finite $p_{\eta}$ modes, the effective momentum of the transverse modes always has a minimum value at $k_{T T}>0$. As a result, fluctuations with higher $p_{T}$ can only have a smaller growth rate. This is the reason why any exponential growth is not clearly identified for these modes.

\section{E. Applicability of the linear analysis}

We discuss the applicability range of the linear analysis that we have adopted. To this end, we focus on the zero momentum mode which shows the strongest instability.

Recalling that the unstable behavior emerges after the transition time $\theta_{\mathrm{tr}}$, the typical time interval $\theta_{\text {lin }}$ in which the linear approximation is valid is estimated as

$$
\theta_{\text {lin }}=\theta_{\text {tr }}+\theta_{\text {grow }}
$$

where $\theta_{\text {grow }}$ is the time scale at which the amplitude of the most unstable mode becomes comparable to that of the background field. As we saw in the previous section, the most unstable mode is the zero momentum mode, and its asymptotic growth rate is well described by that in the nonexpanding geometry. Let us denote the growth rate by $\gamma_{0}$. Then, the time scale $\theta_{\text {grow }}$ is approximately given by

$$
A \sim a_{0} e^{\gamma_{0} \theta_{\text {grow }}} .
$$

Here, $A$ and $a_{0}$ denote the amplitudes of the background field and initial fluctuation. Equations (57) and (58) lead to

$$
\theta_{\mathrm{lin}} \sim \theta_{\mathrm{tr}}+\frac{1}{\gamma_{0}} \log \frac{A}{a_{0}}
$$

If we write the gauge coupling constant $g$ explicitly, the fluctuation amplitude $a$ is initially of order $\mathcal{O}(1)$, and the background field $g A$ is of order $\mathcal{O}(1)$. Thus, the ratio of the amplitude of the background field to that of the initial fluctuation parametrically becomes $A / a_{0} \sim 1 / g$. In the weak coupling regime where, say, $g=10^{-3}$, Eq. (59) reads $\theta_{\text {lin }} \simeq 11$ because of Eqs. (50) and (55).

In Fig. 3, we display the time evolution of fluctuations within the time region $\theta<\theta_{\text {lin. }}$. In this time interval, only $a_{A+}$ and $a_{B-}$ components show instability. We also found that not only low-momentum modes but also highmomentum modes, say $p_{\eta}^{2}=3.0$, show instability. Even when the initial longitudinal momentum is large so that it is located outside the unstable region shown in Fig. 5, it can eventually become small enough to enter the unstable region due to its time dependence. We emphasize that this feature differs from the nonexpanding case.

\section{F. Physical time scale}

Finally, we should estimate the physical scale of the transition times. In order to do that, it is instructive to see the results in the original coordinate instead of the conformal coordinate. We illustrate the numerical solution of $a_{A+}\left(p_{\eta}\right)$ and $a_{1 x}\left(p_{T}\right)$ as a function of the proper time in Fig. 7. Vertical lines indicate the transition times. The time interval shown in Fig. 7 is $0<\tau<\tau_{\operatorname{lin}}=2 \theta_{\operatorname{lin}}^{3 / 2} / 3 \simeq 24.3$, where the linear analysis is valid. We also show the time evolution of the background field in the inset figure for comparison. The time evolution of the unstable modes is well described by the exponential function. For instance, the squared amplitude of $a_{A+}\left(p_{\eta}\right)$ agrees well with $\tau^{-1 / 3} e^{2 \gamma_{0}(3 \tau / 2)^{2 / 3}}$ denoted by the black solid line, where $\gamma_{0} \simeq 0.66$ is the largest growth rate given in Eq. (50). This figure shows that these modes start to grow exponentially in the early stage of the dynamics even in the original coordinate.

As we have seen in Sec. II, all quantities are scaled by the strength of the background magnetic field $B_{\text {eff }}$ in our setup. By using the relation between the conformal and proper times in Eq. (27) and the empirical value of the saturation scale, $Q_{\mathrm{s}} \simeq 1 \mathrm{GeV}$ for the RHIC or $Q_{\mathrm{s}} \simeq 2 \mathrm{GeV}$ for the LHC, the transition time for the fastest growth modes $\left(a_{A+}\right.$ and $\left.a_{B-}\right)$ is estimated as

$$
\begin{aligned}
\tau_{t} & =\frac{2}{3}\left(\theta_{t} B_{\mathrm{eff}}^{1 / 2}\right)^{3 / 2} \frac{1}{Q_{\mathrm{s}}} \\
& \simeq \begin{cases}0.53(1.12) \mathrm{fm} / c & \text { for the RHIC, } \\
0.26(0.56) \mathrm{fm} / c & \text { for the LHC }\end{cases}
\end{aligned}
$$

for $p_{\eta}^{2}=1.0(3.0)$. These fastest growth modes can emerge in the dynamics of relativistic heavy-ion collisions. In heavyion experiments, the rapidity gaps between the projectile and

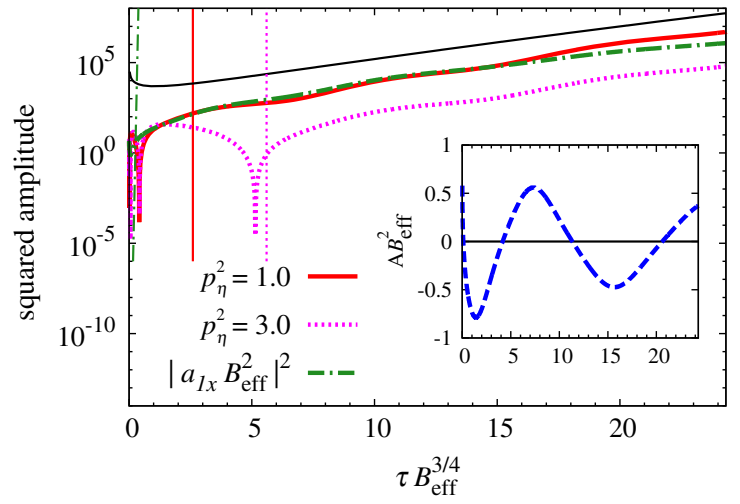

FIG. 7. Squared amplitudes of fluctuations as a function of proper time. Solid line: $a_{A+}$ with $p_{\eta}^{2}=1.0$. Dotted line: $a_{A+}$ with $p_{\eta}^{2}=3.0$. Dashed line: $a_{1 x}$ with $p_{T}^{2}=0.1$. Vertical lines indicate the corresponding transition times shown in Figs. 3 and 4. The Inset figure shows the time evolution of the background field as a function of proper time. 
target are about $\Delta Y=10.7$ in the RHIC and 17.4 in the LHC. The rapidity gap gives the lower bound of the longitudinal momentum of fluctuations; in order to observe half a wavelength of the fluctuation, $\Lambda_{\eta} \Delta Y \sim \pi$ is required. For the RHIC and LHC, we find $\Lambda_{\eta}^{2} \sim 0.08$ and $\Lambda_{\eta}^{2} \sim 0.03$, respectively. The typical momenta of the fastest growth modes, say $p_{\eta}^{2}=1.0(3.0)$, are sufficiently larger than these lower bounds.

We also estimate the physical scale of the instability of finite $p_{T}$ modes. In Fig. 7, the small transverse momentum mode with $p_{T}^{2}=0.1 B_{\text {eff }}^{-3 / 2}$ grows as fast as finite $p_{\eta}$ modes. In the physical unit, this value is estimated as $p_{T} \simeq 0.3 Q_{s} \simeq 0.3-0.6 \mathrm{GeV}$. In heavy-ion collisions, a significant portion of particles are produced in the region $p_{T}<0.3 Q_{s}$, and the instability can be relevant.

These results suggest that the parametric instability might be relevant in the dynamics of CYM field in an expanding geometry.

\section{CONCLUSIONS}

We have studied the instability of the CYM field in an expanding geometry under the longitudinal color magnetic background which is homogeneous, boost invariant, and time dependent. We have introduced the conformal variables and mapped the expanding problem approximately into the nonexpanding problem. Since the background gauge field at later times is described by the elliptic function in the conformal coordinate, we can carry out the stability analysis in a semianalytic way in the expanding geometry on the basis of the Floquet theory in a nonexpanding geometry by introducing the time-dependent effective momenta.

We have performed a linear stability analysis of fluctuations on top of the oscillating background field. We have elucidated that the EOM of fluctuations at later times in the expanding geometry has the same form as in the nonexpanding geometry. At the same time, the EOM in the expanding geometry contains the effective momentum which is a function of the initial momentum and the conformal time. As a result, we have found that the expanding system under the time-dependent background field can show parametric instability, but the instability emerges in a different way from the nonexpanding geometry.

The way of growth is qualitatively different between finite $p_{\eta}$ and finite $p_{T}$ modes. Since the longitudinal effective momentum decreases monotonically, unstable fluctuations with finite $p_{\eta}$ grow exponentially after the transition from the oscillating behavior in the earliest stage. The remarkable point is that not only low-momentum modes but also high-momentum modes show parametric instability. On the other hand, since the transverse effective momentum is not monotonic in time, exponential growth in finite $p_{T}$ modes is limited in a particular temporal interval. In this regime, effective momenta are small and located within the instability band, and then fluctuations can grow exponentially. In accordance with the band structure found in the nonexpanding geometry, only the modes with small $p_{T}$ show significant instability. Finally, we have estimated the physical scale of the transition times. For finite $p_{\eta}$ modes, the typical unstable modes start to grow at $\tau \simeq$ $0.26(0.53) \mathrm{fm} / c$ at LHC (RHIC) energy. These results suggest that the parametric instability could be relevant to the gluodynamics in heavy-ion collisions.

One of the notable points is that the parametric instability emerges at early times in some classes of field theories as discussed, for example, in cosmic inflation [32]. The appearance of the parametric instability seems to be a universal phenomenon in field theories including the YangMills theory. While we have established that the parametric instability emerges under the color magnetic background fields in the homogeneous system, there are still unsolved and interesting questions. One is associated with the robustness of the parametric instability. It is nontrivial whether this instability is relevant or not to inhomogeneous systems, in particular glasma evolution from a more realistic initial condition. It is also important to investigate how the parametric instability affects the particle production where the nonlinear interaction of the gluon fluctuations must be taken into account. Investigations of these problems are beyond the scope of the present work and left as future problems.

\section{ACKNOWLEDGMENTS}

S. T. is supported by the Grant-in-Aid for JSPS fellows (Grant No. 26-3462). This work was supported in part by the Grants-in-Aid for Scientific Research from JSPS (Grants No. 23340067, No. 15K05079, No. 15H03663, and No. 16K05350), the Grants-in-Aid for Scientific Research on Innovative Areas from MEXT (Grants No. 24105001, and No. 24105008), and the Yukawa International Program for Quark-Hadron Sciences.

\section{APPENDIX A: THE LINEARIZED EOM OF FLUCTUATIONS IN AN EXPANDING GEOMETRY}

In this Appendix, we show the explicit form of the linearized EOM of fluctuations in the color magnetic background Eq. (21). The symbolic form of the EOM is given by Eq. (29). Without loss of generality, the coefficient matrix $\Omega^{2}$ is decomposed into two independent sectors $\Omega_{4}^{2}$ and $\Omega_{5}^{2}$ due to the rotational symmetry in the transverse direction. In the momentum representation, the explicit forms of them are given by 
PARAMETRIC INSTABILITY OF CLASSICAL YANG- ...

PHYSICAL REVIEW D 94, 016001 (2016)

$$
\begin{aligned}
\left(\left[\Omega_{4}^{2}\right]_{\alpha \beta}\right) & =\left(\begin{array}{cccc}
\tilde{p}_{x}^{2}+\tilde{\theta}^{-3} \tilde{p}_{\eta}^{2}+\tilde{A}^{2} & 2 \tilde{A}^{2} & 0 & -2 i \tilde{A} \tilde{p}_{x} \\
2 \tilde{A}^{2} & \tilde{\theta}^{-3} \tilde{p}_{\eta}^{2}+\tilde{A}^{2} & -\tilde{\theta}^{-3} \tilde{p}_{\eta} \tilde{p}_{x} & -i \tilde{A} \tilde{p}_{x} \\
0 & -\tilde{\theta}^{-3} \tilde{p}_{\eta} \tilde{p}_{x} & \tilde{\theta}^{-3}\left(\tilde{p}_{x}^{2}+\tilde{A}^{2}\right) & -\tilde{\theta}^{-3} i \tilde{A} \tilde{p}_{\eta} \\
2 i \tilde{A} \tilde{p}_{x} & i \tilde{A} \tilde{p}_{x} & \tilde{\theta}^{-3} i \tilde{A} \tilde{p}_{\eta} & \tilde{p}_{x}^{2}+\tilde{\theta}^{-3} \tilde{p}_{\eta}^{2}+\tilde{A}^{2}
\end{array}\right), \\
\left(\left[\Omega_{5}^{2}\right]_{A B}\right)= & \left(\begin{array}{ccccc}
\tilde{\theta}^{-3} \tilde{p}_{\eta}^{2} & -\tilde{\theta}^{-3} \tilde{p}_{\eta} \tilde{p}_{x} & -\tilde{A}^{2} & 0 & \tilde{\theta}^{-3} i \tilde{A} \tilde{p}_{\eta} \\
-\tilde{\theta}^{-3} \tilde{p}_{\eta} \tilde{p}_{x} & \tilde{\theta}^{-3}\left(\tilde{p}_{x}^{2}+\tilde{A}^{2}\right) & 0 & \tilde{\theta}^{-3} i \tilde{A}_{\eta} \tilde{p}_{\eta} & -\tilde{\theta}^{-3} 2 i \tilde{A} \tilde{p}_{x} \\
-\tilde{A}^{2} & 0 & \tilde{p}_{x}^{2}+\tilde{\theta}^{-3} \tilde{p}_{\eta}^{2} & -i \tilde{A} \tilde{p}_{x} & -\tilde{\theta}^{-3} i \tilde{A} \tilde{p}_{\eta} \\
0 & -\tilde{\theta}^{-3} i \tilde{A} \tilde{p}_{\eta} & i \tilde{A} \tilde{p}_{x} & \tilde{\theta}^{-3} \tilde{p}_{\eta}^{2}+\tilde{A}^{2} & -\tilde{\theta}^{-3} \tilde{p}_{\eta} \tilde{p}_{x} \\
-\tilde{\theta}^{-3} i \tilde{A} \tilde{p}_{\eta} & \tilde{\theta}^{-3} 2 i \tilde{A} \tilde{p}_{x} & \tilde{\theta}^{-3} i \tilde{A} \tilde{p}_{\eta} & -\tilde{\theta}^{-3} \tilde{p}_{\eta} \tilde{p}_{x} & \tilde{\theta}^{-3}\left(\tilde{p}_{x}^{2}+2 \tilde{A}^{2}\right)
\end{array}\right),
\end{aligned}
$$

where $\tilde{p}_{I}=\tilde{\theta}^{1 / 2} p_{I}$ and we use the following notation: $\alpha, \beta, \ldots=(1 y, 2 x, 2 \eta, 3 y)$ and $A, B, \ldots=(1 x, 1 \eta, 2 y, 3 x, 3 \eta)$.

For the sake of the stability analysis performed in Sec. IV, we consider two specific limits $p_{\eta} \neq 0, p_{T}=0$ (finite $p_{\eta}$ modes) and $p_{\eta}=0, p_{T} \neq 0$ (finite $p_{T}$ modes). In these limits, the coefficient matrices (A1) and (A2) are further decomposed to lower rank matrices:

$$
\begin{aligned}
& \Omega_{4}^{2}=\left\{\begin{array}{ll}
\operatorname{diag}\left(\Omega_{B}^{2}, \Omega_{C}^{* 2}\right) & \left(\text { finite } p_{\eta} \text { modes }\right) \\
\operatorname{diag}\left(\Omega_{E}^{2}, \Omega_{G}^{2}\right) & \left(\text { finite } p_{T}\right. \text { modes) }
\end{array},\right. \\
& \Omega_{5}^{2}=\left\{\begin{array}{ll}
\operatorname{diag}\left(\Omega_{A}^{2}, \Omega_{C}^{2}\right) & \left(\text { finite } p_{\eta}\right. \text { modes) } \\
\operatorname{diag}\left(\Omega_{D}^{2}, \Omega_{F}^{2}\right) & \text { (finite } p_{T} \text { modes) }
\end{array} .\right.
\end{aligned}
$$

In the case of finite $p_{\eta}$ modes $\left(p_{\eta} \neq 0, p_{T}=0\right)$, the EOM is decomposed into four independent sectors $A, B, C$, and $C^{*}$. The EOM of the $A$-sector is given by

$$
\begin{gathered}
\ddot{a}_{A+}+k_{\eta T}^{2} a_{A+}-\tilde{A}^{2} a_{A+}=0, \\
\ddot{a}_{A-}+k_{\eta T}^{2} a_{A-}+\tilde{A}^{2} a_{A-}+\sqrt{2} i \tilde{\theta}^{-5 / 2} p_{\eta} \tilde{A} a_{\eta}^{3}=0, \\
\mathcal{L}_{\eta}^{2} a_{\eta}^{3}+M_{\eta}^{2} a_{\eta}^{3}+2 \tilde{A}^{2} a_{\eta}^{3}-\sqrt{2} i \tilde{\theta}^{1 / 2} p_{\eta} \tilde{A} a_{A-}=0, \\
\mathcal{L}_{\eta}^{2}=\frac{d^{2}}{d \theta^{2}}-\frac{2}{\tilde{\theta}} \frac{d}{d \theta},
\end{gathered}
$$

where $a_{A \pm}=\left(a_{x}^{1} \pm a_{y}^{2}\right) / \sqrt{2}, k_{\eta T}^{2}=M_{T}^{2}+9 p_{\eta}^{2} / 4 \theta^{2}$, and $M_{T}^{2}=M_{x}^{2}=M_{y}^{2}=1 / 4 \theta^{2}$. Dots denote derivatives with respect to conformal time $\theta$. The EOM of the $B$-sector reads

$$
\begin{gathered}
\ddot{a}_{B+}+k_{\eta T}^{2} a_{B+}+3 \tilde{A}^{2} a_{B+}=0, \\
\ddot{a}_{B-}+k_{\eta T}^{2} a_{B-}-\tilde{A}^{2} a_{B-}=0,
\end{gathered}
$$

where $a_{B \pm}=\left(a_{y}^{1} \pm a_{x}^{2}\right) / \sqrt{2}$. The EOMs of the $C$ - and $C^{*}$-sectors have the same form: for the $C$-sector,

$$
\begin{gathered}
\ddot{a}_{x}^{3}+k_{\eta T}^{2} a_{x}^{3}+\tilde{A}^{2} a_{x}^{3}-i \tilde{\theta}^{-5 / 2} p_{\eta} \tilde{A} a_{\eta}^{1}=0, \\
\mathcal{L}_{\eta}^{2} a_{\eta}^{1}+M_{\eta}^{2} a_{\eta}^{1}+\tilde{A}^{2} a_{\eta}^{1}+i \tilde{\theta}^{1 / 2} p_{\eta} \tilde{A} a_{x}^{3}=0
\end{gathered}
$$

and for the $C^{*}$-sector,

$$
\begin{gathered}
\ddot{a}_{y}^{3}+k_{\eta T}^{2} a_{y}^{3}+\tilde{A}^{2} a_{y}^{3}+i \tilde{\theta}^{-5 / 2} p_{\eta} \tilde{A} a_{\eta}^{2}=0, \\
\mathcal{L}_{\eta}^{2} a_{\eta}^{2}+M_{\eta}^{2} a_{\eta}^{2}+\tilde{A}^{2} a_{\eta}^{2}-i \tilde{\theta}^{1 / 2} p_{\eta} \tilde{A} a_{y}^{3}=0
\end{gathered}
$$

We note that $M_{\eta}^{2}=7 / 4 \theta^{2}$.

In the case of finite $p_{T}$ modes $\left(p_{\eta}=0, p_{T} \neq 0\right)$, the $\mathrm{EOM}$ is decomposed into four independent sectors $D, E, F$, and $G$. For of the $D$ - and $E$-sectors, we get

$$
\begin{gathered}
\ddot{a}_{x}^{1}+M_{T}^{2} a_{x}^{1}-\tilde{A}^{2} a_{y}^{2}=0, \\
\ddot{a}_{x}^{2}+k_{T T}^{2} a_{y}^{2}-\tilde{A}^{2} a_{x}^{1}-i \tilde{\theta}^{1 / 2} p_{x} \tilde{A} a_{x}^{3}=0, \\
\ddot{a}_{x}^{3}+M_{T}^{2} a_{x}^{3}+\tilde{A}^{2} a_{x}^{3}+i \tilde{\theta}^{1 / 2} p_{x} \tilde{A} a_{y}^{2}=0,
\end{gathered}
$$

and

$$
\begin{gathered}
\ddot{a}_{y}^{1}+k_{T T}^{2} a_{y}^{1}+\tilde{A}^{2} a_{y}^{1}+2 \tilde{A}^{2} a_{x}^{2}-2 i \tilde{\theta}^{1 / 2} p_{x} \tilde{A} a_{y}^{3}=0, \\
\ddot{a}_{y}^{2}+M_{T}^{2}+\tilde{A}^{2} a_{x}^{2}+2 \tilde{A}^{2} a_{y}^{1}-i \tilde{\theta}^{1 / 2} p_{x} \tilde{A} a_{y}^{3}=0, \\
\ddot{a}_{y}^{3}+k_{T T}^{2} a_{y}^{3}+\tilde{A}^{2} a_{y}^{3}+i \tilde{\theta}^{1 / 2} p_{x} \tilde{A}\left(2 a_{y}^{1}+a_{x}^{2}\right)=0,
\end{gathered}
$$

respectively. The EOM of the $F$ - and $G$-sectors is given by

$$
\mathcal{L}_{\eta}^{2} a_{\eta}^{1}+k_{T \eta}^{2} a_{\eta}^{1}+\tilde{A}^{2} a_{\eta}^{1}-2 i \tilde{\theta}^{1 / 2} p_{x} \tilde{A} a_{\eta}^{3}=0,
$$


TSUTSUI, KUNIHIRO, and OHNISHI

$$
\mathcal{L}_{\eta}^{2} a_{\eta}^{3}+k_{T \eta}^{2} a_{\eta}^{3}+2 \tilde{A}^{2} a_{\eta}^{3}+2 i \tilde{\theta}^{1 / 2} p_{x} \tilde{A} a_{\eta}^{1}=0,
$$

and

$$
\mathcal{L}_{\eta}^{2} a_{\eta}^{2}+k_{T \eta}^{2} a_{\eta}^{2}+\tilde{A}^{2} a_{\eta}^{2}=0,
$$

respectively. Here, we use the notion of the effective momenta, $k_{T T}^{2}=M_{T}^{2}+2 \theta p_{x}^{2} / 3$ and $k_{T \eta}^{2}=M_{\eta}^{2}+2 \theta p_{x}^{2} / 3$.

In the following subsections, we give the solutions of the EOM in the early stage $\theta \ll 1$ and the late stage $\theta \gg 1$.

\section{1. $\theta \ll 1$}

Assuming $\theta \ll 1$, the dominant term with respect to $\theta$ is of order $\mathcal{O}\left(\theta^{-2}\right)$. Recalling $\tilde{A} \sim \mathcal{O}\left(\theta^{1 / 2}\right)$, other terms depending on $\theta$ explicitly are of higher order in $\theta$. Collecting leading order terms, we get

$$
\begin{gathered}
\ddot{a}_{A+}+k_{\eta T}^{2} a_{A+}+\mathcal{O}(\theta)=0, \\
\ddot{a}_{A-}+k_{\eta T}^{2} a_{A-}+\mathcal{O}\left(\theta^{-3 / 2}\right)=0, \\
\mathcal{L}_{\eta}^{2} a_{\eta}^{3}+M_{\eta}^{2} a_{\eta}^{3}+\mathcal{O}(\theta)=0
\end{gathered}
$$

for the $A$-sector and

$$
\begin{aligned}
& \ddot{a}_{B+}+k_{\eta T}^{2} a_{B+}+\mathcal{O}(\theta)=0, \\
& \ddot{a}_{B-}+k_{\eta T}^{2} a_{B-}+\mathcal{O}(\theta)=0,
\end{aligned}
$$

for the $B$-sector. The EOMs of the $C$ - and $C^{*}$-sectors are given by

$$
\begin{gathered}
\ddot{a}_{x}^{3}+k_{\eta T}^{2} a_{x}^{3}+\mathcal{O}\left(\theta^{-3 / 2}\right)=0, \\
\mathcal{L}_{\eta}^{2} a_{\eta}^{1}+M_{\eta}^{2} a_{\eta}^{1}+\mathcal{O}(\theta)=0,
\end{gathered}
$$

and

$$
\begin{gathered}
\ddot{a}_{y}^{3}+k_{\eta T}^{2} a_{y}^{3}+\mathcal{O}\left(\theta^{-3 / 2}\right)=0, \\
\mathcal{L}_{\eta}^{2} a_{\eta}^{2}+M_{\eta}^{2} a_{\eta}^{2}+\mathcal{O}(\theta)=0
\end{gathered}
$$

respectively. In summary, when $p_{\eta} \neq 0, p_{T}=0$, all transverse components satisfy

$$
\ddot{a}_{i}^{a}+k_{\eta T}^{2} a_{i}^{a}=0,
$$

while all the longitudinal components obey

$$
\mathcal{L}_{\eta}^{2} a_{\eta}^{a}+M_{\eta}^{2} a_{\eta}^{a}=0 .
$$

PHYSICAL REVIEW D 94, 016001 (2016)

Thus, their general solutions are given by $a_{i}^{a}=$ $c_{1} \theta^{1 / 2+3 i p_{\eta} / 2}+$ c.c. and $a_{\eta}^{a}=c_{1} \theta^{1 / 2}+c_{2} \theta^{7 / 2}$, where $c_{i}$ are arbitrary constants.

In the case of finite $p_{T}$ modes, the $p_{T}$ dependence vanishes for $k_{T T}^{2} \rightarrow M_{T}^{2}$ and $k_{T \eta}^{2} \rightarrow M_{\eta}^{2}$ as $\theta \rightarrow 0$. This leads to

$$
\begin{aligned}
& \ddot{a}_{x}^{1}+M_{T}^{2} a_{x}^{1}+\mathcal{O}(\theta)=0, \\
& \ddot{a}_{y}^{2}+M_{T}^{2} a_{y}^{2}+\mathcal{O}(\theta)=0, \\
& \ddot{a}_{x}^{3}+M_{T}^{2} a_{x}^{3}+\mathcal{O}(\theta)=0
\end{aligned}
$$

for the $D$-sector and

$$
\begin{aligned}
& \ddot{a}_{y}^{1}+M_{T}^{2} a_{y}^{1}+\mathcal{O}(\theta)=0, \\
& \ddot{a}_{x}^{2}+M_{T}^{2} a_{x}^{2}+\mathcal{O}(\theta)=0, \\
& \ddot{a}_{y}^{3}+M_{T}^{2} a_{y}^{3}+\mathcal{O}(\theta)=0
\end{aligned}
$$

for the $E$-sector. We also obtain

$$
\begin{aligned}
& \mathcal{L}_{\eta}^{2} a_{\eta}^{1}+M_{\eta}^{2} a_{\eta}^{1}+\mathcal{O}(\theta)=0, \\
& \mathcal{L}_{\eta}^{2} a_{\eta}^{3}+M_{\eta}^{2} a_{\eta}^{3}+\mathcal{O}(\theta)=0
\end{aligned}
$$

for the $F$-sector and

$$
\mathcal{L}_{\eta}^{2} a_{\eta}^{2}+M_{\eta}^{2} a_{\eta}^{2}+\mathcal{O}(\theta)=0
$$

for the $G$-sector. In summary, when $p_{\eta}=0, p_{T} \neq 0$, all transverse components obey

$$
\ddot{a}_{i}^{a}+M_{T}^{2} a_{i}^{a}=0,
$$

and the general solution is given by $a_{i}^{a}=c_{1} \theta^{1 / 2}+$ $c_{2} \theta^{1 / 2} \log \theta$. The solutions of longitudinal fluctuations are equivalent to the case of $p_{\eta} \neq 0, p_{T}=0$.

\section{2. $\theta \gg 1$}

Assuming that $\theta \gg 1$, terms involving $\theta$ with a negative exponent can be neglected in the first approximation. Recalling that $\tilde{A} \sim \operatorname{cn}(\theta ; 1 / \sqrt{2})$, we can write the EOM in the form

$$
\begin{gathered}
\ddot{a}_{A+}-\mathrm{cn}^{2} \theta a_{A+}=0, \\
\ddot{a}_{A-}+\mathrm{cn}^{2} \theta a_{A-}+\mathcal{O}\left(\theta^{-5 / 2} a_{\eta}^{3}\right)=0, \\
\mathcal{L}_{\eta}^{2} a_{\eta}^{3}+2 \mathrm{cn}^{2} \theta a_{\eta}^{3}-\sqrt{2} i \tilde{\theta}^{1 / 2} p_{\eta} \tilde{A} a_{A-}=0
\end{gathered}
$$

for the $A$-sector and 
PARAMETRIC INSTABILITY OF CLASSICAL YANG- ...

$$
\begin{gathered}
\ddot{a}_{B+}+3 \mathrm{cn}^{2} \theta a_{B+}=0, \\
\ddot{a}_{B-}-\mathrm{cn}^{2} \theta a_{B-}=0
\end{gathered}
$$

for the $B$-sector. The EOMs of the $C$ - and $C^{*}$-sectors are given by

$$
\begin{gathered}
\ddot{a}_{x}^{3}+\operatorname{cn}^{2} \theta a_{x}^{3}+\mathcal{O}\left(\theta^{-5 / 2} a_{\eta}^{1}\right)=0, \\
\mathcal{L}_{\eta}^{2} a_{\eta}^{1}+\operatorname{cn}^{2} \theta a_{\eta}^{1}+i \tilde{\theta}^{1 / 2} p_{\eta} \tilde{A} a_{x}^{3}=0,
\end{gathered}
$$

and

$$
\begin{gathered}
\ddot{a}_{y}^{3}+\operatorname{cn}^{2} \theta a_{y}^{3}+\mathcal{O}\left(\theta^{-5 / 2} a_{\eta}^{2}\right)=0, \\
\mathcal{L}_{\eta}^{2} a_{\eta}^{2}+\operatorname{cn}^{2} \theta a_{\eta}^{2}-i \tilde{\theta}^{1 / 2} p_{\eta} \tilde{A} a_{y}^{3}=0,
\end{gathered}
$$

respectively. Thus, we get Lamé's equations when $p_{\eta} \neq 0$, $p_{T}=0: a_{A+}, a_{B-}$, and $a_{3 \eta}$ have exponential instability and their growth rates are determined by the Floquet theory independent of $p_{\eta}$. It should be noted that $a_{A-}$ has only linear instability in the first approximation, but the last term in Eq. (A46) can be relevant at later times because $a_{\eta}^{3}$ grows exponentially.

On the other hand, the contribution from the background field is washed out for finite $p_{T}$ modes. In the first approximation, the dominant contribution with respect to $\theta$ is of order $\mathcal{O}(\theta)$. The EOMs of the $D$ - and $E$-sectors are given by

$$
\begin{gathered}
\ddot{a}_{x}^{1}-\mathrm{cn}^{2} \theta a_{y}^{2}=0, \\
\ddot{a}_{x}^{2}+2 \theta p_{x}^{2} / 3 a_{y}^{2}+\mathcal{O}\left(\theta^{1 / 2}\right)=0, \\
\ddot{a}_{x}^{3}+\mathcal{O}\left(\theta^{1 / 2}\right)=0
\end{gathered}
$$

and

$$
\begin{gathered}
\ddot{a}_{y}^{1}+2 \theta p_{x}^{2} / 3 a_{y}^{1}+\mathcal{O}\left(\theta^{1 / 2}\right)=0, \\
\ddot{a}_{y}^{2}+\mathcal{O}\left(\theta^{1 / 2}\right)=0, \\
\ddot{a}_{y}^{3}+2 \theta p_{x}^{2} / 3 a_{y}^{3}+\mathcal{O}\left(\theta^{1 / 2}\right)=0,
\end{gathered}
$$

respectively. The EOM of the $F$-sector,

$$
\begin{aligned}
& \mathcal{L}_{\eta}^{2} a_{\eta}^{1}+2 \theta p_{x}^{2} / 3 a_{\eta}^{1}+\mathcal{O}\left(\theta^{1 / 2}\right)=0 \\
& \mathcal{L}_{\eta}^{2} a_{\eta}^{3}+2 \theta p_{x}^{2} / 3 a_{\eta}^{3}+\mathcal{O}\left(\theta^{1 / 2}\right)=0
\end{aligned}
$$

and the EOM of the $G$-sector,

$$
\mathcal{L}_{\eta}^{2} a_{\eta}^{2}+2 \theta p_{x}^{2} / 3 a_{\eta}^{2}=0
$$

PHYSICAL REVIEW D 94, 016001 (2016)

have the same form. In summary, we find that $a_{x}^{2}, a_{y}^{1}$, and $a_{y}^{3}$ obey the Airy equation,

$$
\ddot{a}+\frac{2}{3} \theta p_{x}^{2} a=0
$$

the solution of which is given by $a=$ $c_{1} A i\left(\left(-2 p_{x}^{2} / 3\right)^{1 / 3} \theta\right)+\ldots$. The longitudinal components satisfy the Bessel equation,

$$
\ddot{a}_{\eta}^{a}-\frac{3}{\theta} \dot{a}_{\eta}^{a}+\frac{2}{3} \theta p_{x}^{2} a_{\eta}^{a}=0,
$$

the solution of which is given by $a_{\eta}^{a}=$ $c_{1} \theta^{2} J_{4 / 3}\left(p_{x} \tilde{\theta}^{3 / 2}\right)+\ldots$. Therefore, these modes do not show exponential instability in this stage.

\section{APPENDIX B: LINEAR INSTABILITY}

Floquet's theorem states that the stability of the solution of Lamé's equation (42) is governed by $\operatorname{tr} M$. If $|\operatorname{tr} M|=2$, there is not only stable solution but also linear divergent solution. Here, we show the origin of the linear divergence. Suppose that $\mu_{1}$ and $\mu_{2}$ are the eigenvalues of $M$; the condition $|\operatorname{tr} M|=2$ is realized if and only if $\mu_{1}=\mu_{2}= \pm 1$. The monodromy matrix cannot be semisimple but only has the Jordan normal form due to the degeneracy of the characteristic multiplier. If $\operatorname{tr} M=2$, without loss of generality, we can take

$$
M=\left(\begin{array}{ll}
1 & 1 \\
0 & 1
\end{array}\right)
$$

The logarithm of $M$ is now given by

$$
\log M=\log \left[1+\left(\begin{array}{ll}
0 & 1 \\
0 & 0
\end{array}\right)\right]=\left(\begin{array}{ll}
0 & 1 \\
0 & 0
\end{array}\right) .
$$

Finally, we get

$$
\Phi(t)=F(t) \exp \left(\begin{array}{cc}
0 & t / T \\
0 & 0
\end{array}\right)=F(t)\left(\begin{array}{cc}
1 & t / T \\
0 & 1
\end{array}\right),
$$

where $F(t)$ is a periodic function. Actually, one solution is periodic in time, and the another solution is linearly divergent. The case of $\operatorname{tr} M=-2$ can be discussed in a parallel way, and then we get antiperiodic and linearly divergent solutions. In general, the degeneracy occurs at the boundaries of the instability bands.

\section{APPENDIX C: EFFECTIVE GROWTH RATE}

In Sec. IV, we have shown that the growth rates of finite $p_{\eta}$ modes approach the upper bound which is determined by the Floquet analysis in a nonexpanding geometry. More quantitative discussion can be performed as follows. 
TSUTSUI, KUNIHIRO, and OHNISHI

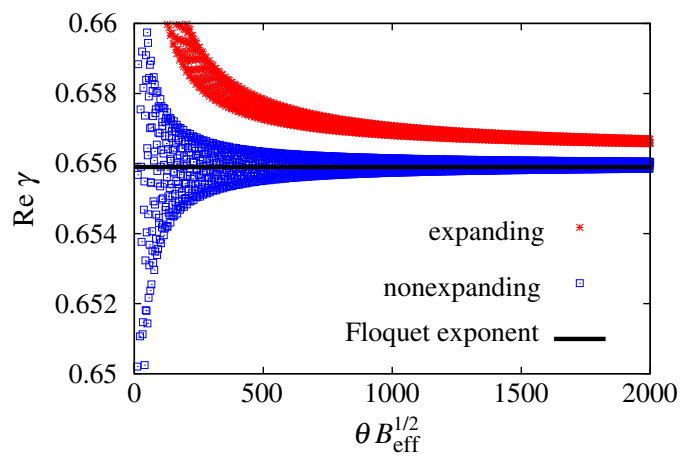

FIG. 8. The effective growth rate of the $a_{A+}(p=0)$ (expanding) and Lamé's equation with $\lambda=-1$ (nonexpanding). The black solid line describes the Floquet exponent.

Suppose that $\Phi(\theta)$ is a Wronskian matrix of the given second order differential equation, and let us define
PHYSICAL REVIEW D 94, 016001 (2016)

$M\left(\theta_{0}, \theta\right)=\Phi\left(\theta_{0}\right)^{-1} \Phi(\theta)$.

$M\left(\theta_{0}, T+\theta_{0}\right)$ is equivalent to the monodromy matrix for the periodic-driven system with period $T$. Thus, the straightforward generalization of the Floquet exponents are given by the eigenvalues of the $\Gamma(\theta)=\log M /\left(\theta-\theta_{0}\right)$, say $\left\{\gamma_{i}\right\}$. We call them effective growth rates.

We calculate the maximal effective growth rate for $a_{A+}(p=0)$ and Lamé's equation with $\lambda=-1$ which is the nonexpanding counterpart. Figure 8 shows the real part of the effective growth rate as a function of the conformal time. The effective growth rate of the Lamé equation agrees well with the Floquet exponent of which the value is approximately 0.6559 as it should be. The effective growth rate of $a_{A+}(p=0)$ is slightly larger than that of Lamé's equation, but it approaches the Floquet exponent.

[19] E. S. Weibel, Phys. Rev. Lett. 2, 83 (1959).

[20] S. Mrowczynski, Phys. Lett. B 214, 587 (1988).

[1] U. W. Heinz, AIP Conf. Proc. 739, 163 (2005).

[2] D. A. Teaney, arXiv:0905.2433.

[3] T. Hirano, P. Huovinen, K. Murase, and Y. Nara, Prog. Part. Nucl. Phys. 70, 108 (2013).

[4] R. Baier, A. H. Mueller, D. Schiff, and D. Son, Phys. Lett. B 502, 51 (2001).

[5] R. Baier, A. H. Mueller, D. Schiff, and D. Son, Phys. Lett. B 539, 46 (2002).

[6] L. D. McLerran and R. Venugopalan, Phys. Rev. D 49, 2233 (1994).

[7] F. Gelis, E. Iancu, J. Jalilian-Marian, and R. Venugopalan, Annu. Rev. Nucl. Part. Sci. 60, 463 (2010).

[8] T. Lappi and L. McLerran, Nucl. Phys. A772, 200 (2006).

[9] J. Berges, S. Scheffler, and D. Sexty, Phys. Lett. B 681, 362 (2009).

[10] T. Kunihiro, B. Muller, A. Ohnishi, and A. Schafer, Prog. Theor. Phys. 121, 555 (2009).

[11] T. Kunihiro, B. Muller, A. Ohnishi, A. Schafer, T. T. Takahashi, and A. Yamamoto, Phys. Rev. D 82, 114015 (2010).

[12] H. Iida, T. Kunihiro, B. Muller, A. Ohnishi, A. Schafer, and T. T. Takahashi, Phys. Rev. D 88, 094006 (2013).

[13] P. Romatschke and R. Venugopalan, Phys. Rev. Lett. 96, 062302 (2006).

[14] P. Romatschke and R. Venugopalan, Phys. Rev. D 74, 045011 (2006).

[15] J. Berges, S. Scheffler, and D. Sexty, Phys. Rev. D 77, 034504 (2008).

[16] T. Epelbaum and F. Gelis, Nucl. Phys. A926, 122 (2014).

[17] A. Rebhan, M. Strickland, and M. Attems, Phys. Rev. D 78, 045023 (2008).

[18] M. Attems, A. Rebhan, and M. Strickland, Phys. Rev. D 87, 025010 (2013).

[21] P. B. Arnold, J. Lenaghan, and G. D. Moore, J. High Energy Phys. 08 (2003) 002.

[22] P. B. Arnold, J. Lenaghan, G. D. Moore, and L. G. Yaffe, Phys. Rev. Lett. 94, 072302 (2005).

[23] N. Nielsen and P. Olesen, Nucl. Phys. B144, 376 (1978).

[24] H. Fujii and K. Itakura, Nucl. Phys. A809, 88 (2008).

[25] H. Fujii, K. Itakura, and A. Iwazaki, Nucl. Phys. A828, 178 (2009).

[26] A. Iwazaki, Prog. Theor. Phys. 121, 809 (2009).

[27] A. Kurkela and G. D. Moore, J. High Energy Phys. 12 (2011) 044.

[28] A. Kurkela and G. D. Moore, J. High Energy Phys. 11 (2011) 120.

[29] J. Berges, S. Scheffler, S. Schlichting, and D. Sexty, Phys. Rev. D 85, 034507 (2012).

[30] S. Tsutsui, H. Iida, T. Kunihiro, and A. Ohnishi, Phys. Rev. D 91, 076003 (2015).

[31] J. Berges and J. Serreau, Phys. Rev. Lett. 91, 111601 (2003).

[32] M. A. Amin, M. P. Hertzberg, D. I. Kaiser, and J. Karouby, Int. J. Mod. Phys. D 24, 1530003 (2015).

[33] J. Berges, K. Boguslavski, and S. Schlichting, Phys. Rev. D 85, 076005 (2012).

[34] K. Dusling, T. Epelbaum, F. Gelis, and R. Venugopalan, Phys. Rev. D 86, 085040 (2012).

[35] J. D. Bjorken, Phys. Rev. D 27, 140 (1983).

[36] Essentially, the amplitude of the fluctuations should be much smaller than the amplitude of the background field. However, the amplitudes are irrelevant to our discussion since we employ the linear approximation. 